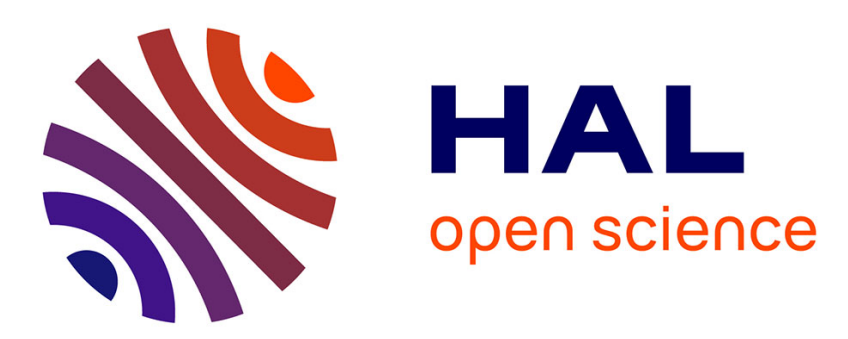

\title{
A unified framework for a posteriori error estimation for the Stokes problem
}

\author{
Antti Hannukainen, Rolf Stenberg, Martin Vohralík
}

\section{To cite this version:}

Antti Hannukainen, Rolf Stenberg, Martin Vohralík. A unified framework for a posteriori error estimation for the Stokes problem. Numerische Mathematik, 2012, 122 (4), pp.725-769. 10.1007/s00211012-0472-x . hal-00470131v2

\section{HAL Id: hal-00470131 \\ https://hal.science/hal-00470131v2}

Submitted on 28 Jan 2011

HAL is a multi-disciplinary open access archive for the deposit and dissemination of scientific research documents, whether they are published or not. The documents may come from teaching and research institutions in France or abroad, or from public or private research centers.
L'archive ouverte pluridisciplinaire $\mathbf{H A L}$, est destinée au dépôt et à la diffusion de documents scientifiques de niveau recherche, publiés ou non, émanant des établissements d'enseignement et de recherche français ou étrangers, des laboratoires publics ou privés. 
Antti Hannukainen • Rolf Stenberg •

Martin Vohralík

\title{
A unified framework for a posteriori error estimation for the Stokes problem
}

Received: date / Revised: date

\begin{abstract}
In this paper, a unified framework for a posteriori error estimation for the Stokes problem is developed. It is based on $\left[H_{0}^{1}(\Omega)\right]^{d}$-conforming velocity reconstruction and $\underline{\boldsymbol{H}}(\operatorname{div}, \Omega)$-conforming, locally conservative flux (stress) reconstruction. It gives guaranteed, fully computable global upper bounds as well as local lower bounds on the energy error. In order to apply this framework to a given numerical method, two simple conditions need to be checked. We show how to do this for various conforming and conforming stabilized finite element methods, the discontinuous Galerkin method, the Crouzeix-Raviart nonconforming finite element method, the mixed finite element method, and a general class of finite volume methods. Numerical experiments illustrate the theoretical developments.
\end{abstract}

Keywords Stokes problem · a posteriori error estimate $\cdot$ unified framework · finite element method · discontinuous Galerkin method · mixed finite element method $\cdot$ finite volume method

Mathematics Subject Classification (2000) 65N15, 76M12, 76S05

This work was supported by the GNR MoMaS project "Numerical Simulations and Mathematical Modeling of Underground Nuclear Waste Disposal", PACEN/CNRS, ANDRA, BRGM, CEA, EdF, IRSN, France, the Finnish Research Programme on Nuclear Waste Management (KYT), and the Academy of Finland (decision number 133174

A. Hannukainen · R. Stenberg

Department of Mathematics and Systems Analysis, Aalto University, P.O. Box 11100, 00076 Aalto, Finland

E-mail: antti.hannukainen@tkk.fi, rolf.stenberg@tkk.fi

M. Vohralík

UPMC Univ. Paris 06, UMR 7598, Laboratoire Jacques-Louis Lions, 75005, Paris, France

\&

CNRS, UMR 7598, Laboratoire Jacques-Louis Lions, 75005, Paris, France

E-mail: vohralik@ann.jussieu.fr 


\section{Introduction}

The purpose of this paper is to develop a unified framework for a posteriori error estimation for the Stokes problem discretized by different numerical methods. In particular, we apply this framework to conforming divergencefree, discontinuous Galerkin, conforming (stabilized), nonconforming, mixed, and finite volume methods. Our estimates give a guaranteed (that is, not featuring any undetermined constant) upper bound on the error measured in the energy (semi-)norm, provided a bound on the inf-sup constant is known. They are easily, fully, and locally computable. They are also locally efficient in the sense that they represent local lower bounds for the energy error. Numerical experiments show that their effectivity index (the ratio of the estimated and exact error) is relatively close to the optimal value of one.

Our estimates are based on $\left[H_{0}^{1}(\Omega)\right]^{d}$-conforming velocity reconstruction and $\underline{\boldsymbol{H}}(\operatorname{div}, \Omega)$-conforming, locally conservative flux (stress) reconstruction. Such an approach has recently become popular in the framework of secondorder elliptic equations, see, e.g., [44,39,50,4,27,45,2,43,59,3,31,60] and the references cited therein. Its main ideas are very physical and can be traced back at least to the Prager-Synge equality [49]. Equilibrated flux estimates have recently been shown to be robust with respect to inhomogeneities, anisotropies, and reaction or convection dominance in $[63,24,32]$ and with respect to the approximation polynomial degree in [13]. In a unifying spirit, similar to the present paper, they have been extended to the heat equation in [34]. Stokes a posterior error estimates related to the present approach have previously been studied in $[29,51,10]$. However, these estimates are valid only for certain type of numerical approximations. Here, we develop a unified framework covering most standard methods. The technique and proofs that we use appear to be new for the Stokes problem, and as they are nontrivial generalizations of the corresponding techniques for the Poisson problem, we present them in all details. For the classical residual-based estimates presented in a unified framework, we refer to [20,23,22] and [64].

Locally conservative $\underline{\boldsymbol{H}}(\operatorname{div}, \Omega)$-conforming flux reconstruction is straightforward in so-called locally conservative methods $[2,43,59,3,31,61,33,32,62$, 34]. For finite element-type methods, which are not locally conservative by construction, this is less straightforward. However, for such methods, the reconstruction can be achieved by the equilibration procedure, see $[4,27,13]$ and the references therein. We follow here the approach for lowest-order methods of $[45,60,63]$, where no equilibration is needed. We generalize this approach here to higher-order methods. It turns out that only small local problems of fixed size $(d+1) \times(d+1)$ for each mesh element, where $d$ is the space dimension, need to be solved in order to obtain the equilibrated side normal fluxes.

This paper is organized as follows. In Section 2, we state the considered Stokes problem. In Section 3, we specify our notation and give some preliminary results. Sections 4 and 5 collect our a posteriori error estimates, first for conforming divergence-free approximations and then for arbitrary ones. These results are stated in a general form independent of the numerical method at hand; we only suppose the existence of a locally conservative $\underline{\boldsymbol{H}}(\operatorname{div}, \Omega)$-conforming flux reconstruction $\underline{\boldsymbol{\sigma}}_{h}$ (cf. assumptions (4.3) and (5.9) below). Section 6 then presents the efficiency of the estimates, still in a general form independent of the numerical method at hand, only based on Assumption 6.2. In Section 7, we apply the previous results to different numerical methods. This consists in specifying the way of construction of $\underline{\sigma}_{h}$ and in verifying the assumptions (4.3) or (5.9) and Assumption 6.2. Section 8 col- 
lects some numerical experiments. Appendix A gives a useful characterization of the inf-sup constants and Appendix B presents the equilibration-type procedure generalizing the dual mesh-based a posteriori error estimates to higher-order conforming and conforming stabilized finite element methods.

\section{The Stokes problem}

Here, we describe the Stokes problem considered in this paper. We use standard notation; some details on the notation are given in Section 3 below.

Let $\Omega \subset \mathbb{R}^{d}, d=2,3$, be a polygonal (polyhedral) domain (open, bounded, and connected set). We consider the Stokes problem: given $\mathbf{f} \in\left[L^{2}(\Omega)\right]^{d}$, find $\mathbf{u}$, the "velocity", and $p$, the "pressure", such that

$$
\begin{array}{rll}
-\Delta \mathbf{u}+\nabla p=\mathbf{f} & \text { in } \quad \Omega, \\
\nabla \cdot \mathbf{u}=0 & \text { in } \quad \Omega, \\
\mathbf{u}=\mathbf{0} & \text { on } \quad \partial \Omega .
\end{array}
$$

Denote by $\mathbf{V}$ the space $\left[H_{0}^{1}(\Omega)\right]^{d}$ and by $Q$ the space of $L^{2}(\Omega)$ functions having zero mean value over $\Omega$. For $\mathbf{u}, \mathbf{v} \in \mathbf{V}$ and $q \in Q$, set

$$
\begin{aligned}
a(\mathbf{u}, \mathbf{v}) & :=(\nabla \mathbf{u}, \nabla \mathbf{v}), \\
b(\mathbf{v}, q) & :=-(q, \nabla \cdot \mathbf{v}) .
\end{aligned}
$$

The weak formulation of (2.1a)-(2.1c) reads: find $(\mathbf{u}, p) \in \mathbf{V} \times Q$ such that

$$
\begin{aligned}
a(\mathbf{u}, \mathbf{v})+b(\mathbf{v}, p) & =(\mathbf{f}, \mathbf{v}) & & \forall \mathbf{v} \in \mathbf{V}, \\
b(\mathbf{u}, q) & =0 & & \forall q \in Q .
\end{aligned}
$$

The above problem is well-posed (cf. [37]) due to the inf-sup condition

$$
\inf _{q \in Q} \sup _{\mathbf{v} \in \mathbf{V}} \frac{b(\mathbf{v}, q)}{\|\nabla \mathbf{v}\|\|q\|}=\beta
$$

where $\beta$ is a positive constant. Denote the divergence-free subspace of $\mathbf{V}$ by

$$
\mathbf{V}_{0}:=\{\mathbf{v} \in \mathbf{V} ; \nabla \cdot \mathbf{v}=0\}
$$

The velocity $\mathbf{u}$ can be equivalently characterized as: find $\mathbf{u} \in \mathbf{V}_{0}$ such that

$$
a(\mathbf{u}, \mathbf{v})=(\mathbf{f}, \mathbf{v}) \quad \forall \mathbf{v} \in \mathbf{V}_{0} .
$$

Recall also that by introducing the "stress" tensor $\underline{\boldsymbol{\sigma}} \in \underline{\boldsymbol{H}}(\operatorname{div}, \Omega)$, the problem (2.1a)-(2.1c) can be written as a system consisting of the constitutive law

the equilibrium equation

$$
\underline{\boldsymbol{\sigma}}=\nabla \mathbf{u}-p \underline{\boldsymbol{I}}
$$

$$
\nabla \cdot \underline{\boldsymbol{\sigma}}+\mathbf{f}=\mathbf{0}
$$

and the divergence constraint

$$
\nabla \cdot \mathbf{u}=0
$$

for which the pressure $p$ is the Lagrange multiplier. Here $\underline{\boldsymbol{I}}$ is the $d \times d$ identity matrix. Alternatively, (2.6)-(2.7) may be replaced by

$$
\underline{\boldsymbol{\sigma}}^{\prime}=\nabla \mathbf{u}
$$

and

$$
\nabla \cdot \underline{\boldsymbol{\sigma}}^{\prime}-\nabla p+\mathbf{f}=\mathbf{0}
$$




\section{Notation and preliminaries}

Here, we summarize the notation used throughout the paper and give some preliminary results.

\subsection{Notation}

Let $D \subset \mathbb{R}^{d}$. By $(\cdot, \cdot)_{D}$, we denote the scalar product in $L^{2}(D):(p, q)_{D}:=$ $\int_{D} p q \mathrm{~d} \mathbf{x}$. When $D$ coincides with $\Omega$, the subscript $\Omega$ will be dropped. We use the same symbol $(\cdot, \cdot)_{D}$ for the scalar product in $\mathbf{L}^{2}(D):=\left[L^{2}(D)\right]^{d}$ and in $\underline{\boldsymbol{L}}^{2}(D):=\left[L^{2}(D)\right]^{d \times d}$. More precisely, $(\mathbf{u}, \mathbf{v})_{D}:=\sum_{i=1}^{d}\left(\mathbf{u}^{i}, \mathbf{v}^{i}\right)_{D}$ for $\mathbf{u}, \mathbf{v} \in \mathbf{L}^{2}(D)$ and $(\underline{\boldsymbol{\sigma}}, \boldsymbol{\tau})_{D}:=\sum_{i=1}^{d} \sum_{j=1}^{d}\left(\underline{\boldsymbol{\sigma}}^{i, j}, \underline{\boldsymbol{\tau}}^{i, j}\right)_{D}$ for $\underline{\boldsymbol{\sigma}}, \underline{\boldsymbol{\tau}} \in \underline{\boldsymbol{L}}^{2}(D)$. The associated norm is denoted by $\|\cdot\|_{D}$. We denote by $\langle\cdot, \cdot\rangle$ the scalar product in $L^{2}(D), D \subset \mathbb{R}^{d-1}$, and its vector and tensor versions. For vectors $\mathbf{u}, \mathbf{v} \in \mathbb{R}^{d}$, $\mathbf{u} \otimes \mathbf{v}$ defines a tensor $\underline{\boldsymbol{\sigma}} \in \mathbb{R}^{d \times d}$ such that $\underline{\boldsymbol{\sigma}}^{i, j}:=\mathbf{u}^{i} \mathbf{v}^{j}$. Finally, for $D \subset \mathbb{R}^{d^{\prime}}$, $1 \leq d^{\prime} \leq d,|D|$ stands for the $d^{\prime}$-dimensional Lebesgue measure of $D$ and we denote by $\mathbf{e}_{i} \in \mathbb{R}^{d}$ the $i$-th Euclidean unit vector.

Let $\mathcal{T}_{h}$ be a polygonal (polyhedral) partition of $\Omega$, whose elements can be nonconvex or non star-shaped. The partition $\mathcal{T}_{h}$ can be nonmatching, that is, the intersection of two elements $T, T^{\prime}$ of $\mathcal{T}_{h}$ is not necessarily their common face, edge, or vertex or an empty set (so-called hanging nodes are allowed). We denote by $h_{T}$ the diameter of $T \in \mathcal{T}_{h}$. We say that $F$ is an interior side of $\mathcal{T}_{h}$ if it has a positive $(d-1)$-dimensional Lebesgue measure and if there are distinct $T^{-}(F)$ and $T^{+}(F)$ in $\mathcal{T}_{h}$ such that $F=\partial T^{-}(F) \cap \partial T^{+}(F)$. We define $\mathbf{n}_{F}$ as the unit normal vector to $F$ pointing from $T^{-}(F)$ towards $T^{+}(F)$. Similarly, we say that $F$ is a boundary side of $\mathcal{T}_{h}$ if it has a positive $(d-1)$-dimensional Lebesgue measure and if there is $T(F) \in \mathcal{T}_{h}$ such that $F=\partial T(F) \cap \partial \Omega$ and we define $\mathbf{n}_{F}$ as the unit outward normal to $\partial \Omega$. The arbitrariness in the orientation of $\mathbf{n}_{F}$ is irrelevant in the sequel. All the interior (resp., boundary) sides of the mesh are collected into the set $\partial \mathcal{T}_{h}^{\text {int }}$ (resp., $\partial \mathcal{T}_{h}^{\text {ext }}$ ) and we set $\partial \mathcal{T}_{h}:=\partial \mathcal{T}_{h}^{\text {int }} \cup \partial \mathcal{T}_{h}^{\text {ext }}$. For $F \in \partial \mathcal{T}_{h}, h_{F}$ stands for its diameter. For $T \in \mathcal{T}_{h}$, we denote by $\mathcal{F}_{T}$ all its sides and by $\mathcal{F}_{T}^{\text {int }}$ those sides of $T$ which belong to $\partial \mathcal{T}_{h}^{\text {int }}$. We will also use the notation $\mathfrak{T}_{T}$ (resp., $\mathfrak{F}_{T}$ ) for the elements (resp., sides) of $\mathcal{T}_{h}$ sharing a vertex with $T$. We denote by $\mathfrak{F}_{T}^{\text {int }}$ those sides of $\mathfrak{F}_{T}$ which belong to $\partial \mathcal{T}_{h}^{\text {int }}$. The notation $\mathcal{V}_{h}\left(\right.$ resp., $\left.\mathcal{V}_{h}^{\text {int }}\right)$ will be used for the set of all (resp., interior) vertices of $\mathcal{T}_{h}$. Let $V \in \mathcal{V}_{h}$. Then $\mathfrak{T}_{V}$ denotes all the elements of $\mathcal{T}_{h}$ having $V$ as vertex.

For a (sufficiently smooth) scalar, vector, or tensor function $v$ that is double-valued on an interior side $F$, its jump and average on $F$ are defined as

$$
\llbracket v \rrbracket_{F}:=\left.v\right|_{T^{-}(F)}-\left.v\right|_{T^{+}(F)}, \quad\left\{\{v\}_{F}:=\frac{1}{2}\left(\left.v\right|_{T^{-}(F)}+\left.v\right|_{T^{+}(F)}\right) .\right.
$$

We set $\llbracket v \rrbracket_{F}:=\left.v\right|_{F}$ and $\{v v\}_{F}:=\left.v\right|_{F}$ on boundary sides. The subscript $F$ in the above jumps and averages is omitted if there is no ambiguity. We denote by $\mathbf{V}\left(\mathcal{T}_{h}\right)$ the space of piecewise smooth vector functions on $\mathcal{T}_{h}$

$$
\mathbf{V}\left(\mathcal{T}_{h}\right):=\left\{\mathbf{v}_{h} \in \mathbf{L}^{2}(\Omega) ;\left.\mathbf{v}_{h}\right|_{T} \in\left[H^{1}(T)\right]^{d} \quad \forall T \in \mathcal{T}_{h}\right\}
$$

Note that $\mathbf{V}\left(\mathcal{T}_{h}\right) \not \subset \mathbf{V}$. We employ the notation $\mathbb{P}_{k}\left(\mathcal{T}_{h}\right)$ for piecewise polynomials of order $k$ on $\mathcal{T}_{h}$. In the sequel, we use the signs $\nabla, \Delta$, and $\nabla$. respectively for the elementwise gradient, Laplace, and divergence operators. Some additional notation will also be introduced later where needed. 


\subsection{Preliminaries}

Let $T \in \mathcal{T}_{h}$ and denote by $\boldsymbol{\varphi}_{T}$ the average of $\boldsymbol{\varphi}$ over $T$, i.e., $\boldsymbol{\varphi}_{T}^{i}=\left(\boldsymbol{\varphi}, \mathbf{e}_{i}\right)_{T} /|T|$, $i=1, \ldots, d$. Then the Poincaré inequality states

$$
\left\|\boldsymbol{\varphi}-\boldsymbol{\varphi}_{T}\right\|_{T} \leq C_{\mathrm{P}, T} h_{T}\|\nabla \boldsymbol{\varphi}\|_{T} \quad \forall \boldsymbol{\varphi} \in\left[H^{1}(T)\right]^{d},
$$

where the constant $C_{\mathrm{P}, T}$ is independent of $h_{T}$. It depends only on the shape of $T$. For a convex $T$, we have the estimate $C_{\mathrm{P}, T} \leq 1 / \pi[47,9]$.

Set

$$
\mathcal{B}((\mathbf{v}, q),(\mathbf{z}, r)):=a(\mathbf{v}, \mathbf{z})+b(\mathbf{z}, q)+b(\mathbf{v}, r) .
$$

The problem (2.3a)-(2.3b) can then be stated as: find $(\mathbf{u}, p) \in \mathbf{V} \times Q$ such that

$$
\mathcal{B}((\mathbf{u}, p),(\mathbf{v}, q))=(\mathbf{f}, \mathbf{v}) \quad \forall(\mathbf{v}, q) \in \mathbf{V} \times Q .
$$

We define the energy (semi-)norm for $(\mathbf{v}, q) \in \mathbf{V}\left(\mathcal{T}_{h}\right) \times Q$ as

$$
\|(\mathbf{v}, q)\|^{2}:=\|\nabla \mathbf{v}\|^{2}+\beta^{2}\|q\|^{2},
$$

where $\beta$ is the constant from the inf-sup condition (2.4). The following stability estimate has been communicated to us by J.-F. Maître [46]:

Lemma 3.1 (The inf-sup condition on $\mathbf{V} \times Q$ ) There is a positive constant $C_{\mathrm{S}}$ such that

$$
\inf _{(\mathbf{v}, q) \in \mathbf{V} \times Q} \sup _{(\mathbf{z}, r) \in \mathbf{V} \times Q} \frac{\mathcal{B}((\mathbf{v}, q),(\mathbf{z}, r))}{\|(\mathbf{z}, r)\| \mid\|(\mathbf{v}, q)\|}=C_{\mathrm{S}}
$$

with

$$
C_{\mathrm{S}}=\frac{\sqrt{5}-1}{2}
$$

Note that $1 / C_{\mathrm{S}}=(\sqrt{5}+1) / 2$, which is the golden ratio. For the sake of completeness, we give a proof of Lemma 3.1 in Appendix A below.

\section{A posteriori error estimate for conforming divergence-free approximations}

In this section, we derive an a posteriori error estimate valid for arbitrary conforming and divergence-free approximations, i.e., with velocities $\mathbf{u}_{h} \in \mathbf{V}_{0}$. It can be considered as an intermediate result, as standard approximation methods do not lead to $\mathbf{u}_{h} \in \mathbf{V}_{0}$. There exist, however, methods fulfilling this constraint, like that of [52].

Given an approximation $\left(\mathbf{u}_{h}, p_{h}\right) \in \mathbf{V}_{0} \times Q$, not necessarily the numerical solution, the a posteriori error estimators on $T \in \mathcal{T}_{h}$ are defined as follows. Let $\underline{\boldsymbol{\sigma}}_{h} \in \underline{\boldsymbol{H}}(\operatorname{div}, \Omega)$. We define the residual estimator

$$
\eta_{\mathrm{R}, T}:=C_{\mathrm{P}, T} h_{T}\left\|\nabla \cdot \underline{\boldsymbol{\sigma}}_{h}+\mathbf{f}\right\|_{T},
$$

where $C_{\mathrm{P}, T}$ is the constant from the Poincaré inequality (3.2), and the diffusive flux estimator

$$
\eta_{\mathrm{DF}, T}:=\left\|\nabla \mathbf{u}_{h}-p_{h} \underline{\boldsymbol{I}}-\underline{\boldsymbol{\sigma}}_{h}\right\|_{T} .
$$

We then have the following estimate. 
Theorem 4.1 (Velocity estimate for conforming divergence-free approximations.) Let $\mathbf{u} \in \mathbf{V}_{0}$ be the weak solution given by (2.5) and let $\left(\mathbf{u}_{h}, p_{h}\right) \in \mathbf{V}_{0} \times Q$ be arbitrary. Let $\underline{\boldsymbol{\sigma}}_{h} \in \underline{\boldsymbol{H}}(\operatorname{div}, \Omega)$ be such that

$$
\left(\nabla \cdot \underline{\boldsymbol{\sigma}}_{h}+\mathbf{f}, \mathbf{e}_{i}\right)_{T}=0, \quad i=1, \ldots, d, \quad \forall T \in \mathcal{T}_{h}
$$

Then

$$
\left\|\nabla\left(\mathbf{u}-\mathbf{u}_{h}\right)\right\| \leq\left\{\sum_{T \in \mathcal{T}_{h}}\left(\eta_{\mathrm{R}, T}+\eta_{\mathrm{DF}, T}\right)^{2}\right\}^{1 / 2} .
$$

Proof Using (2.2a) and (2.5), we have

$$
\begin{aligned}
\left\|\nabla\left(\mathbf{u}-\mathbf{u}_{h}\right)\right\| & =a\left(\mathbf{u}-\mathbf{u}_{h}, \frac{\mathbf{u}-\mathbf{u}_{h}}{\left\|\nabla\left(\mathbf{u}-\mathbf{u}_{h}\right)\right\|}\right) \\
& \leq \sup _{\boldsymbol{\varphi} \in \mathbf{V}_{0}} \frac{a\left(\mathbf{u}-\mathbf{u}_{h}, \boldsymbol{\varphi}\right)}{\|\nabla \boldsymbol{\varphi}\|} \\
& =\sup _{\boldsymbol{\varphi} \in \mathbf{V}_{0}} \frac{(\mathbf{f}, \boldsymbol{\varphi})-a\left(\mathbf{u}_{h}, \boldsymbol{\varphi}\right)}{\|\nabla \boldsymbol{\varphi}\|} .
\end{aligned}
$$

Let $\varphi \in \mathbf{V}_{0}$ be fixed. Then, using that $\nabla \cdot \varphi=0$,

$$
0=\left(p_{h}, \nabla \cdot \boldsymbol{\varphi}\right)=\left(p_{h} \underline{\boldsymbol{I}}, \nabla \boldsymbol{\varphi}\right) .
$$

Moreover, using the Green theorem $\left(\underline{\sigma}_{h}, \nabla \boldsymbol{\varphi}\right)=-\left(\nabla \cdot \underline{\sigma}_{h}, \varphi\right)$ and adding and subtracting $\left(\underline{\sigma}_{h}, \nabla \varphi\right)$,

$$
\begin{aligned}
& (\mathbf{f}, \boldsymbol{\varphi})-a\left(\mathbf{u}_{h}, \boldsymbol{\varphi}\right) \\
& \quad=(\mathbf{f}, \boldsymbol{\varphi})-\left(\nabla \mathbf{u}_{h}, \nabla \boldsymbol{\varphi}\right)+\left(p_{h} \underline{\boldsymbol{I}}, \nabla \boldsymbol{\varphi}\right)+\left(\underline{\boldsymbol{\sigma}}_{h}, \nabla \boldsymbol{\varphi}\right)-\left(\underline{\boldsymbol{\sigma}}_{h}, \nabla \boldsymbol{\varphi}\right) \\
& \quad=\left(\mathbf{f}+\nabla \cdot \underline{\boldsymbol{\sigma}}_{h}, \boldsymbol{\varphi}\right)-\left(\nabla \mathbf{u}_{h}-p_{h} \underline{\boldsymbol{I}}-\underline{\boldsymbol{\sigma}}_{h}, \nabla \boldsymbol{\varphi}\right) .
\end{aligned}
$$

Let $T \in \mathcal{T}_{h}$. Then, using the assumption (4.3), the Cauchy-Schwarz inequality, the Poincaré inequality (3.2), and the definition (4.1), we get

$$
\left(\nabla \cdot \underline{\boldsymbol{\sigma}}_{h}+\mathbf{f}, \boldsymbol{\varphi}\right)_{T}=\left(\nabla \cdot \underline{\boldsymbol{\sigma}}_{h}+\mathbf{f}, \boldsymbol{\varphi}-\boldsymbol{\varphi}_{T}\right)_{T} \leq \eta_{\mathrm{R}, T}\|\nabla \boldsymbol{\varphi}\|_{T} .
$$

Next, the estimate

$$
\left(\nabla \mathbf{u}_{h}-p_{h} \underline{\boldsymbol{I}}-\underline{\boldsymbol{\sigma}}_{h}, \nabla \boldsymbol{\varphi}\right)_{T} \leq \eta_{\mathrm{DF}, T}\|\nabla \boldsymbol{\varphi}\|_{T}
$$

is immediate by the Cauchy-Schwarz inequality and the definition (4.2). The above developments give

$$
\left\|\nabla\left(\mathbf{u}-\mathbf{u}_{h}\right)\right\| \leq \sup _{\boldsymbol{\varphi} \in \mathbf{V}_{0}} \frac{\sum_{T \in \mathcal{T}_{h}}\left\{\left(\eta_{\mathrm{R}, T}+\eta_{\mathrm{DF}, T}\right)\|\nabla \varphi\|_{T}\right\}}{\|\nabla \varphi\|},
$$

whence (4.4) follows by the Cauchy-Schwarz inequality.

Theorem 5.1 below, with $\mathbf{s}_{h}=\mathbf{u}_{h}$, gives the following additional result to Theorem 4.1:

Corollary 4.1 (Pressure estimate for conforming divergence-free approximations) Let $(\mathbf{u}, p) \in \mathbf{V} \times Q$ be the weak solution given by (2.3a)(2.3b). Further, let $\left(\mathbf{u}_{h}, p_{h}\right) \in \mathbf{V}_{0} \times Q$ be arbitrary. Assume that $\underline{\boldsymbol{\sigma}}_{h} \in$ $\underline{\boldsymbol{H}}(\operatorname{div}, \Omega)$ satisfies (4.3). Then it holds

$$
\beta\left\|p-p_{h}\right\| \leq \frac{1}{C_{\mathrm{S}}}\left\{\sum_{T \in \mathcal{T}_{h}}\left(\eta_{\mathrm{R}, T}+\eta_{\mathrm{DF}, T}\right)^{2}\right\}^{1 / 2} .
$$




\section{A posteriori error estimate for general approximations}

In this section we derive our main a posteriori error estimate. This estimate is valid for an approximation $\left(\mathbf{u}_{h}, p_{h}\right) \in \mathbf{V}\left(\mathcal{T}_{h}\right) \times Q$, not necessarily the numerical solution. Note that the approximate velocity $\mathbf{u}_{h}$ can be nonconforming and non-divergence-free.

The a posteriori error estimators on $T \in \mathcal{T}_{h}$ are defined as follows. The possible nonconformity of $\mathbf{u}_{h}$, i.e., the fact that $\mathbf{u}_{h}$ is not necessarily in $\mathbf{V}$, is estimated by the nonconformity estimator

$$
\eta_{\mathrm{NC}, T}:=\left\|\nabla\left(\mathbf{u}_{h}-\mathbf{s}_{h}\right)\right\|_{T}
$$

where $\mathbf{s}_{h} \in \mathbf{V}$ is arbitrary. Next, the divergence estimator, related to the divergence-free constraint (2.8), is given by

$$
\eta_{\mathrm{D}, T}:=\frac{\left\|\nabla \cdot \mathbf{s}_{h}\right\|_{T}}{\beta} .
$$

As in Section 4, the key for our a posteriori error estimates is to construct a flux (stress field) $\underline{\boldsymbol{\sigma}}_{h} \in \underline{\boldsymbol{H}}(\mathrm{div}, \Omega)$ that is in approximate local equilibrium, i.e., satisfying (4.3). It enters in the residual estimator

$$
\eta_{\mathrm{R}, T}:=C_{\mathrm{P}, T} h_{T}\left\|\nabla \cdot \underline{\boldsymbol{\sigma}}_{h}+\mathbf{f}\right\|_{T},
$$

related to the possible violation of the equilibrium equation (2.7) in the approximate solution (here $C_{\mathrm{P}, T}$ is the constant from the Poincaré inequality (3.2)), and in the diffusive flux estimator

$$
\eta_{\mathrm{DF}, T}:=\left\|\nabla \mathbf{s}_{h}-p_{h} \underline{\boldsymbol{I}}-\underline{\boldsymbol{\sigma}}_{h}\right\|_{T},
$$

related to the fact that the constitutive law (2.6) is not satisfied exactly by the approximate solution. Recall the definition (3.5) of the energy (semi-)norm.

Our main theorem is the following.

Theorem 5.1 (Estimate for general approximations) Let $(\mathbf{u}, p) \in \mathbf{V} \times$ $Q$ be the weak solution given by (2.3a)-(2.3b) and let $\left(\mathbf{u}_{h}, p_{h}\right) \in \mathbf{V}\left(\mathcal{T}_{h}\right) \times$ $Q$ be arbitrary. Choose an arbitrary $\mathbf{s}_{h} \in \mathbf{V}$ and $\underline{\boldsymbol{\sigma}}_{h} \in \underline{\boldsymbol{H}}(\operatorname{div}, \Omega)$ which satisfies (4.3). Then it holds

$$
\begin{aligned}
& \|\|\left(\mathbf{u}-\mathbf{u}_{h}, p-p_{h}\right) \mid \| \\
& \quad \leq\left\{\sum_{T \in \mathcal{T}_{h}} \eta_{\mathrm{NC}, T}^{2}\right\}^{1 / 2}+\frac{1}{C_{\mathrm{S}}}\left\{\sum_{T \in \mathcal{T}_{h}}\left\{\left(\eta_{\mathrm{R}, T}+\eta_{\mathrm{DF}, T}\right)^{2}+\eta_{\mathrm{D}, T}^{2}\right\}\right\}^{1 / 2} .
\end{aligned}
$$

Proof By the triangle inequality we have

$$
\left\|\left(\mathbf{u}-\mathbf{u}_{h}, p-p_{h}\right)\right\| \mid \leq\left\|\nabla\left(\mathbf{u}_{h}-\mathbf{s}_{h}\right)\right\|+\left\|\left(\mathbf{u}-\mathbf{s}_{h}, p-p_{h}\right)\right\| .
$$

Using the stability estimate (3.6) (note that $\mathbf{u}-\mathbf{s}_{h} \in \mathbf{V}$ ), we obtain

$$
\left\|\left(\mathbf{u}-\mathbf{s}_{h}, p-p_{h}\right) \mid\right\| \leq \frac{1}{C_{\mathrm{S}}} \sup _{(\boldsymbol{\varphi}, \psi) \in \mathbf{V} \times Q} \frac{\mathcal{B}\left(\left(\mathbf{u}-\mathbf{s}_{h}, p-p_{h}\right),(\boldsymbol{\varphi}, \psi)\right)}{\|(\boldsymbol{\varphi}, \psi)\|} .
$$

Let $(\boldsymbol{\varphi}, \psi) \in \mathbf{V} \times Q$ be fixed. Employing the definitions (3.3) and (3.4), we have

$$
\begin{aligned}
& \mathcal{B}\left(\left(\mathbf{u}-\mathbf{s}_{h}, p-p_{h}\right),(\boldsymbol{\varphi}, \psi)\right) \\
& \quad=\mathcal{B}((\mathbf{u}, p),(\boldsymbol{\varphi}, \psi))-\mathcal{B}\left(\left(\mathbf{s}_{h}, p_{h}\right),(\boldsymbol{\varphi}, \psi)\right) \\
& \quad=(\mathbf{f}, \boldsymbol{\varphi})-\left(\nabla \mathbf{s}_{h}, \nabla \boldsymbol{\varphi}\right)+\left(\nabla \cdot \boldsymbol{\varphi}, p_{h}\right)+\left(\nabla \cdot \mathbf{s}_{h}, \psi\right) .
\end{aligned}
$$


Next, using that $\left(p_{h}, \nabla \cdot \boldsymbol{\varphi}\right)=\left(p_{h} \underline{\boldsymbol{I}}, \nabla \boldsymbol{\varphi}\right)$, adding and subtracting $\left(\underline{\boldsymbol{\sigma}}_{h}, \nabla \boldsymbol{\varphi}\right)$, and using the Green theorem, we get

$$
\begin{aligned}
\mathcal{B} & \left(\left(\mathbf{u}-\mathbf{s}_{h}, p-p_{h}\right),(\boldsymbol{\varphi}, \psi)\right) \\
& =(\mathbf{f}, \boldsymbol{\varphi})-\left(\nabla \mathbf{s}_{h}, \nabla \boldsymbol{\varphi}\right)+\left(p_{h} \underline{\boldsymbol{I}}, \nabla \boldsymbol{\varphi}\right)+\left(\nabla \cdot \mathbf{s}_{h}, \psi\right)+\left(\underline{\boldsymbol{\sigma}}_{h}, \nabla \boldsymbol{\varphi}\right)-\left(\underline{\boldsymbol{\sigma}}_{h}, \nabla \boldsymbol{\varphi}\right) \\
& =\left(\nabla \cdot \underline{\boldsymbol{\sigma}}_{h}+\mathbf{f}, \boldsymbol{\varphi}\right)-\left(\nabla \mathbf{s}_{h}-p_{h} \underline{\boldsymbol{I}}-\underline{\boldsymbol{\sigma}}_{h}, \nabla \boldsymbol{\varphi}\right)+\left(\nabla \cdot \mathbf{s}_{h}, \psi\right) .
\end{aligned}
$$

We estimate the first two terms as in the proof of Theorem 4.1, using the equilibrium condition (4.3) and the Poincaré inequality (3.2). For the last term, we use the Cauchy-Schwarz inequality to obtain

$$
\begin{aligned}
\mathcal{B} & \left(\left(\mathbf{u}-\mathbf{s}_{h}, p-p_{h}\right),(\boldsymbol{\varphi}, \psi)\right) \\
& \leq \sum_{T \in \mathcal{T}_{h}}\left(\eta_{\mathrm{R}, T}+\eta_{\mathrm{DF}, T}\right)\|\nabla \boldsymbol{\varphi}\|_{T}+\frac{\beta}{\beta}\left\|\nabla \cdot \mathbf{s}_{h}\right\|\|\psi\| \\
& \leq\left\{\sum_{T \in \mathcal{T}_{h}}\left\{\left(\eta_{\mathrm{R}, T}+\eta_{\mathrm{DF}, T}\right)^{2}+\eta_{\mathrm{D}, T}^{2}\right\}\right\}^{1 / 2}\|(\boldsymbol{\varphi}, \psi)\| .
\end{aligned}
$$

The assertion then follows by collecting the above estimates.

Let, for $T \in \mathcal{T}_{h}, \eta_{\mathrm{NC}, T}$ and $\eta_{\mathrm{D}, T}$ by given respectively by (5.1) and (5.2) and set

$$
\eta_{\mathrm{R}, T}:=C_{\mathrm{P}, T} h_{T}\left\|\nabla \cdot \underline{\boldsymbol{\sigma}}_{h}-\nabla p_{h}+\mathbf{f}\right\|_{T}
$$

and

$$
\eta_{\mathrm{DF}, T}:=\left\|\nabla \mathbf{s}_{h}-\underline{\boldsymbol{\sigma}}_{h}\right\|_{T} .
$$

In the sequel, we will also need the following modified version of Theorem 5.1.

Corollary 5.1 (An alternative version of Theorem 5.1) Let $(\mathbf{u}, p) \in$ $\mathbf{V} \times Q$ be the weak solution given by $(2.3 \mathrm{a})-(2.3 \mathrm{~b})$ and let $\left(\mathbf{u}_{h}, p_{h}\right) \in \mathbf{V}\left(\mathcal{T}_{h}\right) \times$ $\left[Q \cap H^{1}(\Omega)\right]$ be arbitrary. Choose an arbitrary $\mathbf{s}_{h} \in \mathbf{V}$ and $\underline{\boldsymbol{\sigma}}_{h} \in \underline{\boldsymbol{H}}(\operatorname{div}, \Omega)$ such that

$$
\left(\nabla \cdot \underline{\boldsymbol{\sigma}}_{h}-\nabla p_{h}+\mathbf{f}, \mathbf{e}_{i}\right)_{T}=0, \quad i=1, \ldots, d, \quad \forall T \in \mathcal{T}_{h} .
$$

Then it holds

$$
\begin{aligned}
& \left\|\left(\mathbf{u}-\mathbf{u}_{h}, p-p_{h}\right)\right\| \\
& \quad \leq\left\{\sum_{T \in \mathcal{T}_{h}} \eta_{\mathrm{NC}, T}^{2}\right\}^{1 / 2}+\frac{1}{C_{\mathrm{S}}}\left\{\sum_{T \in \mathcal{T}_{h}}\left\{\left(\eta_{\mathrm{R}, T}+\eta_{\mathrm{DF}, T}\right)^{2}+\eta_{\mathrm{D}, T}^{2}\right\}\right\}^{1 / 2} .
\end{aligned}
$$

Proof We proceed as in the proof of Theorem 5.1 ; only the term $\left(\nabla \cdot \varphi, p_{h}\right)$ in (5.6) is treated differently. By the assumption $p_{h} \in H^{1}(\Omega)$ and the Green theorem, we get $\left(\nabla \cdot \boldsymbol{\varphi}, p_{h}\right)=-\left(\nabla p_{h}, \boldsymbol{\varphi}\right)$. The rest of the proof follows easily while using assumption (5.9) instead of (4.3).

Remark 5.1 (Equilibrated flux $\underline{\boldsymbol{\sigma}}_{h}$ ) The equilibrated flux $\underline{\boldsymbol{\sigma}}_{h}$ in Theorems 4.1 and 5.1 and in Corollary 4.1 is a $\underline{\boldsymbol{H}}(\operatorname{div}, \Omega)$-conforming reconstruction of the flux $\nabla \mathbf{u}_{h}-p_{h} \underline{\boldsymbol{I}}$. It is related to the decomposition (2.6)-(2.7). It will typically apply to such numerical methods where $p_{h} \notin H^{1}(\Omega)$. The equilibrated flux $\underline{\boldsymbol{\sigma}}_{h}$ in Corollary 5.1 is instead a $\underline{\boldsymbol{H}}(\operatorname{div}, \Omega)$-conforming reconstruction of the flux $\nabla \mathbf{u}_{h}$. It is related to the decomposition (2.9)-(2.10). It will typically apply to such numerical methods where $p_{h} \in H^{1}(\Omega)$. 


\section{Local efficiencies}

In this section, we prove the local efficiencies of the estimates introduced above.

First, we make the following assumption. Note that this assumption is only needed in this section.

Assumption 6.1 (Local efficiency) We suppose that, for some $k \geq 1$,

- $\mathbf{u}_{h} \in\left[\mathbb{P}_{k}\left(\mathcal{T}_{h}\right)\right]^{d}, p_{h} \in \mathbb{P}_{k}\left(\mathcal{T}_{h}\right)$, and $\mathbf{f} \in\left[\mathbb{P}_{k}\left(\mathcal{T}_{h}\right)\right]^{d}$,

- there exists a shape-regular matching simplicial submesh $\mathcal{S}_{h}$ of $\mathcal{T}_{h}$,

- for each $T \in \mathcal{T}_{h}$, the number of subelements $T^{\prime} \subset T, T^{\prime} \in \mathcal{S}_{h}$, is uniformly bounded

- the reconstructed flux $\underline{\boldsymbol{\sigma}}_{h} \in\left[\mathbb{P}_{k}\left(\mathcal{S}_{h}\right)\right]^{d \times d}$.

When $\mathcal{T}_{h}$ is itself simplicial and matching, we will in many cases simply use $\mathcal{S}_{h}=\mathcal{T}_{h}$. A mesh $\mathcal{S}_{h} \neq \mathcal{T}_{h}$ will be needed for conforming methods or when $\mathcal{T}_{h}$ is not a simplicial mesh or is nonmatching.

We next introduce some new notation. We use $A \lesssim B$ when there exists a positive constant $C$, independent of the mesh size, of $\Omega$, and of $\mathbf{u}$ and $p$ but dependent on the space dimension $d$, on the shape regularity parameter of the mesh $\mathcal{S}_{h}$, and on the maximal polynomial degree $k$, such that $A \leq C B$.

In order to proceed without specifying a particular numerical method, we will now make an additional assumption. In Section 7 below, this assumption will be verified for the methods in question. Recall that for $T \in \mathcal{T}_{h}$, the classical local residual error indicator (cf. $[56,57,26,40])$ writes

$$
\begin{aligned}
\eta_{\mathrm{res}, T}^{2}:= & \sum_{T \in \mathfrak{T}_{T}}\left\{h_{T}^{2}\left\|\mathbf{f}+\Delta \mathbf{u}_{h}-\nabla p_{h}\right\|_{T}^{2}+\left\|\nabla \cdot \mathbf{u}_{h}\right\|_{T}^{2}\right\} \\
& +\sum_{F \in \mathfrak{F}_{T}^{\text {int }}} h_{F}\left\|\llbracket\left(\nabla \mathbf{u}_{h}-p_{h} \underline{\boldsymbol{I}}\right) \mathbf{n}_{F} \rrbracket\right\|_{F}^{2}+\sum_{F \in \mathfrak{F}_{T}} h_{F}^{-1}\left\|\llbracket \mathbf{u}_{h} \rrbracket\right\|_{F}^{2} .
\end{aligned}
$$

We assume that a quantity linked to our estimators $\eta_{\mathrm{DF}, T}$ is a local lower bound for $\eta_{\mathrm{res}, T}$ :

Assumption 6.2 (Approximation property) For all $T \in \mathcal{T}_{h}$, there holds

$$
\left\|\nabla \mathbf{u}_{h}-p_{h} \underline{\boldsymbol{I}}-\underline{\boldsymbol{\sigma}}_{h}\right\|_{T} \lesssim \eta_{\mathrm{res}, T}
$$

in the case where $\underline{\boldsymbol{\sigma}}_{h}$ satisfies (4.3) and

$$
\left\|\nabla \mathbf{u}_{h}-\underline{\boldsymbol{\sigma}}_{h}\right\|_{T} \lesssim \eta_{\mathrm{res}, T}
$$

in the case where $\underline{\boldsymbol{\sigma}}_{h}$ satisfies (5.9).

By $\mathcal{I}_{\text {av }}:\left[\mathbb{P}_{k}\left(\mathcal{S}_{h}\right)\right]^{d} \rightarrow\left[\mathbb{P}_{k}\left(\mathcal{S}_{h}\right)\right]^{d} \cap \mathbf{V}$, we denote the following averaging operator: let $\mathbf{v}_{h} \in\left[\mathbb{P}_{k}\left(\mathcal{S}_{h}\right)\right]^{d}$. Then $\mathcal{I}_{\text {av }}$ prescribes the Lagrangian degrees of freedom of $\mathcal{I}_{\text {av }}\left(\mathbf{v}_{h}\right) \in\left[\mathbb{P}_{k}\left(\mathcal{S}_{h}\right)\right]^{d} \cap \mathbf{V}$ inside $\Omega$ by the average of the values of $\mathbf{v}_{h}$ and sets $\mathbf{0}$ on $\partial \Omega$. For the analysis we need the following result $[1,42,19$, $61]$.

Lemma 6.3 (Averaging approximation estimate) For $\mathbf{s}_{h}=\mathcal{I}_{\text {av }}\left(\mathbf{u}_{h}\right)$, there holds, for all $T \in \mathcal{T}_{h}$,

$$
\begin{aligned}
\left\|\nabla\left(\mathbf{u}_{h}-\mathbf{s}_{h}\right)\right\|_{T} & \lesssim\left\{\sum_{F \in \mathfrak{F}_{T}} h_{F}^{-1}\left\|\llbracket \mathbf{u}_{h} \rrbracket\right\|_{F}^{2}\right\}^{1 / 2} \\
\left\|\mathbf{u}_{h}-\mathbf{s}_{h}\right\|_{T} & \lesssim\left\{\sum_{F \in \mathfrak{F}_{T}} h_{F}\left\|\llbracket \mathbf{u}_{h} \rrbracket\right\|_{F}^{2}\right\}^{1 / 2} .
\end{aligned}
$$


We now state and prove the main result of this section.

Theorem 6.1 (Local efficiency) Let Assumptions 6.1 and 6.2 be satisfied. Let $\mathbf{s}_{h}=\mathcal{I}_{\mathrm{av}}\left(\mathbf{u}_{h}\right)$ and let, for $T \in \mathcal{T}_{h}$, any of the following possibilities hold:

- $\eta_{\mathrm{R}, T}$ and $\eta_{\mathrm{DF}, T}$ are given by (4.1)-(4.2),

- $\eta_{\mathrm{NC}, T}, \eta_{\mathrm{D}, T}, \eta_{\mathrm{R}, T}$, and $\eta_{\mathrm{DF}, T}$ are given by $(5.1)-(5.4)$,

- $\eta_{\mathrm{NC}, T}$ and $\eta_{\mathrm{D}, T}$ are given by $(5.1)-(5.2)$ and $\eta_{\mathrm{R}, T}$ and $\eta_{\mathrm{DF}, T}$ are given by $(5.7)-(5.8)$.

Let finally $(\mathbf{u}, p) \in \mathbf{V} \times Q$ be the weak solution given by (2.3a)-(2.3b). Then it holds

$$
\eta_{T} \lesssim\left\|\left|\left(\mathbf{u}-\mathbf{u}_{h}, p-p_{h}\right) \|\right|_{\mathfrak{T}_{T}}\right.
$$

for all the local estimators $\eta_{T}=\eta_{\mathrm{NC}, T}, \eta_{\mathrm{D}, T}, \eta_{\mathrm{R}, T}$, and $\eta_{\mathrm{DF}, T}$.

Proof Let $T \in \mathcal{T}_{h}$. We will first bound the individual estimators by $\eta_{\mathrm{res}, T}$ or by its components.

For $\eta_{\mathrm{DF}, T}$ given by (4.2), we have $\eta_{\mathrm{DF}, T} \lesssim \eta_{\mathrm{res}, T}$ by Assumption 6.2 . For $\eta_{\mathrm{DF}, T}$ given by (5.4), the triangle inequality gives

$$
\eta_{\mathrm{DF}, T} \leq\left\|\nabla \mathbf{s}_{h}-\nabla \mathbf{u}_{h}\right\|_{T}+\left\|\nabla \mathbf{u}_{h}-p_{h} \underline{\boldsymbol{I}}-\underline{\boldsymbol{\sigma}}_{h}\right\|_{T},
$$

whence $\eta_{\mathrm{DF}, T} \lesssim \eta_{\text {res,T }}$ by combining Assumption 6.2 and (6.4a). For the third alternative, $\eta_{\mathrm{DF}, T}$ given by (5.8), using the triangle inequality,

$$
\eta_{\mathrm{DF}, T} \leq\left\|\nabla \mathbf{s}_{h}-\nabla \mathbf{u}_{h}\right\|_{T}+\left\|\nabla \mathbf{u}_{h}-\underline{\boldsymbol{\sigma}}_{h}\right\|_{T},
$$

whence once again $\eta_{\mathrm{DF}, T} \lesssim \eta_{\mathrm{res}, T}$ by Assumption 6.2 and (6.4a).

The estimator $\eta_{\mathrm{NC}, T}$ is bounded directly by $(6.4 \mathrm{a})$.

For the estimator $\eta_{\mathrm{R}, T}$ of (4.1), we have

$$
\begin{aligned}
\eta_{\mathrm{R}, T} & \lesssim h_{T}\left\|\mathbf{f}+\Delta \mathbf{u}_{h}-\nabla p_{h}\right\|_{T}+h_{T}\left\|-\Delta \mathbf{u}_{h}+\nabla p_{h}+\nabla \cdot \underline{\boldsymbol{\sigma}}_{h}\right\|_{T} \\
& =h_{T}\left\|\mathbf{f}+\Delta \mathbf{u}_{h}-\nabla p_{h}\right\|_{T}+h_{T}\left\|\nabla \cdot\left(\nabla \mathbf{u}_{h}-p_{h} \underline{\boldsymbol{I}}-\underline{\boldsymbol{\sigma}}_{h}\right)\right\|_{T} \\
& \lesssim h_{T}\left\|\mathbf{f}+\Delta \mathbf{u}_{h}-\nabla p_{h}\right\|_{T}+\left\|\nabla \mathbf{u}_{h}-p_{h} \underline{\boldsymbol{I}}-\underline{\boldsymbol{\sigma}}_{h}\right\|_{T}
\end{aligned}
$$

by the triangle inequality and by the inverse inequality. The bound $\eta_{\mathrm{R}, T} \lesssim$ $\eta_{\mathrm{res}, T}$ thus follows by Assumption 6.2 . For $\eta_{\mathrm{R}, T}$ given by (5.7), we similarly have

$$
\begin{aligned}
\eta_{\mathrm{R}, T} & \lesssim h_{T}\left\|\mathbf{f}+\Delta \mathbf{u}_{h}-\nabla p_{h}\right\|_{T}+h_{T}\left\|-\Delta \mathbf{u}_{h}+\nabla \cdot \underline{\boldsymbol{\sigma}}_{h}\right\|_{T} \\
& \lesssim h_{T}\left\|\mathbf{f}+\Delta \mathbf{u}_{h}-\nabla p_{h}\right\|_{T}+\left\|\nabla \mathbf{u}_{h}-\underline{\boldsymbol{\sigma}}_{h}\right\|_{T},
\end{aligned}
$$

whence $\eta_{\mathrm{R}, T} \lesssim \eta_{\mathrm{res}, T}$ by Assumption 6.2 .

We are left with bounding $\eta_{\mathrm{D}, T}$. We have

$$
\eta_{\mathrm{D}, T} \leq \frac{1}{\beta}\left(\left\|\nabla \cdot\left(\mathbf{s}_{h}-\mathbf{u}_{h}\right)\right\|_{T}+\left\|\nabla \cdot \mathbf{u}_{h}\right\|_{T}\right) \lesssim \frac{1}{\beta}\left(h_{T}^{-1}\left\|\mathbf{s}_{h}-\mathbf{u}_{h}\right\|_{T}+\left\|\nabla \cdot \mathbf{u}_{h}\right\|_{T}\right),
$$

whence, by $(6.4 \mathrm{~b}), \eta_{\mathrm{D}, T} \lesssim \eta_{\mathrm{res}, T}$.

We have now bounded all the local error indicators by $\eta_{\mathrm{res}, T}$. The assertion of the theorem follows by the fact that this classical residual a posteriori error estimate is a lower bound for the energy error, see $[56,57,26,21]$ (the term $\left\{\sum_{F \in \mathfrak{F}_{T}} h_{F}^{-1}\left\|\llbracket \mathbf{u}_{h} \rrbracket\right\|_{F}^{2}\right\}^{1 / 2}$ is bounded in [21, Theorem 5.5]). 


\section{Application to different numerical methods}

In this section, we derive a posteriori error estimates for different numerical methods using Theorem 4.1, Corollary 4.1, Theorem 5.1, or Corollary 5.1. This consists in specifying a way for constructing the flux $\underline{\boldsymbol{\sigma}}_{h} \in \underline{\boldsymbol{H}}(\operatorname{div}, \Omega)$ satisfying (4.3) or (5.9). Remark that this construction is always local. We also check, via Theorem 6.1, that the local efficiency holds for the derived estimates. This consists in verifying Assumption 6.2.

\subsection{Discontinuous Galerkin method}

We apply here Theorems 5.1 and 6.1 for deriving locally efficient a posteriori error estimates for the discontinuous Galerkin method. For simplicity, we suppose that $\mathcal{T}_{h}$ consists of simplices and is matching. The straightforward modifications to general meshes $\mathcal{T}_{h}$ can be carried out along the lines of [33] or [32, Appendix].

Define

$$
\begin{aligned}
\mathbf{V}_{h} & :=\left[\mathbb{P}_{k}\left(\mathcal{T}_{h}\right)\right]^{d}, \\
Q_{h} & :=\mathbb{P}_{k-1}\left(\mathcal{T}_{h}\right) \cap Q,
\end{aligned}
$$

$k \geq 1$. Next, set

$$
\begin{aligned}
a_{h}\left(\mathbf{u}_{h}, \mathbf{v}_{h}\right):= & \sum_{T \in \mathcal{T}_{h}}\left(\nabla \mathbf{u}_{h}, \nabla \mathbf{v}_{h}\right)_{T}+\sum_{F \in \partial \mathcal{T}_{h}} \gamma_{F} h_{F}^{-1}\left\langle\llbracket \mathbf{u}_{h} \rrbracket, \llbracket \mathbf{v}_{h} \rrbracket\right\rangle_{F} \\
& -\sum_{F \in \partial \mathcal{T}_{h}}\left\{\left\langle\left\{\left\{\nabla \mathbf{u}_{h}\right\}\right\} \mathbf{n}_{F}, \llbracket \mathbf{v}_{h} \rrbracket\right\rangle_{F}+\theta\left\langle\left\{\left\langle\nabla \mathbf{v}_{h}\right\}\right\} \mathbf{n}_{F}, \llbracket \mathbf{u}_{h} \rrbracket\right\rangle_{F}\right\}
\end{aligned}
$$

and

$$
b_{h}\left(\mathbf{v}_{h}, q_{h}\right):=-\sum_{T \in \mathcal{T}_{h}}\left(q_{h}, \nabla \cdot \mathbf{v}_{h}\right)_{T}+\sum_{F \in \partial \mathcal{T}_{h}}\left\langle\left\{\left\{q_{h}\right\}\right\}, \llbracket \mathbf{v}_{h} \rrbracket \cdot \mathbf{n}_{F}\right\rangle_{F} .
$$

Here, $\gamma_{F}>0, F \in \partial \mathcal{T}_{h}$, is a parameter (chosen sufficiency large), and $\theta=$ $\{-1,0,1\}$. The discontinuous Galerkin method for the problem (2.3a)-(2.3b) reads: find $\left(\mathbf{u}_{h}, p_{h}\right) \in \mathbf{V}_{h} \times Q_{h}$ such that

$$
\begin{aligned}
a_{h}\left(\mathbf{u}_{h}, \mathbf{v}_{h}\right)+b_{h}\left(\mathbf{v}_{h}, p_{h}\right) & =\left(\mathbf{f}, \mathbf{v}_{h}\right) & & \forall \mathbf{v}_{h} \in \mathbf{V}_{h}, \\
b_{h}\left(\mathbf{u}_{h}, q_{h}\right) & =0 & & \forall q_{h} \in Q_{h} .
\end{aligned}
$$

We now specify $\underline{\boldsymbol{\sigma}}_{h} \in \underline{\boldsymbol{H}}(\operatorname{div}, \Omega)$ satisfying (4.3). We follow [43,31] in the second-order elliptic setting. For a recent similar reconstruction for the Stokes problem, we refer to [10]. Our postprocessed flux $\underline{\boldsymbol{\sigma}}_{h}$ will belong to the Raviart-Thomas-Nédélec space of tensor functions,

$$
\underline{\boldsymbol{\Sigma}}^{l}\left(\mathcal{T}_{h}\right)=\left\{\underline{\boldsymbol{v}}_{h} \in \underline{\boldsymbol{H}}(\operatorname{div}, \Omega) ;\left.\underline{\boldsymbol{v}}_{h}\right|_{T} \in \underline{\boldsymbol{\Sigma}}^{l}(T) \quad \forall T \in \mathcal{T}_{h}\right\},
$$

where $l$ is either $k-1$ or $k$ and

$$
\underline{\boldsymbol{\Sigma}}^{l}(T)=\left[\mathbb{P}_{l}(T)\right]^{d \times d}+\left[\mathbb{P}_{l}(T)\right]^{d} \otimes \mathbf{x} .
$$

In particular, $\underline{\boldsymbol{v}}_{h} \in \underline{\boldsymbol{\Sigma}}^{l}\left(\mathcal{T}_{h}\right)$ is such that $\left.\nabla \cdot \underline{\boldsymbol{v}}_{h}\right|_{T} \in\left[\mathbb{P}_{l}(T)\right]^{d}$ for all $T \in \mathcal{T}_{h}$, $\underline{\boldsymbol{v}}_{h} \mathbf{n}_{F} \in\left[\mathbb{P}_{l}(F)\right]^{d}$ for all $F \in \mathcal{F}_{T}$ and all $T \in \mathcal{T}_{h}$, and such that its normal trace is continuous, cf. [17]. 
We prescribe $\underline{\boldsymbol{\sigma}}_{h} \in \underline{\boldsymbol{\Sigma}}^{l}\left(\mathcal{T}_{h}\right)$ locally on all $T \in \mathcal{T}_{h}$ as follows: for all $F \in \mathcal{F}_{T}$ and all $\mathbf{q}_{h} \in\left[\mathbb{P}_{l}(\bar{F})\right]^{d}$,

$$
\left\langle\underline{\boldsymbol{\sigma}}_{h} \mathbf{n}_{F}, \mathbf{q}_{h}\right\rangle_{F}=\left\langle\left\{\left\{\nabla \mathbf{u}_{h}-p_{h} \underline{\boldsymbol{I}}\right\} \mathbf{n}_{F}-\gamma_{F} h_{F}^{-1} \llbracket \mathbf{u}_{h} \rrbracket, \mathbf{q}_{h}\right\rangle_{F},\right.
$$

and for all $\underline{\boldsymbol{\tau}}_{h} \in\left[\mathbb{P}_{l-1}(T)\right]^{d \times d}$,

$$
\left(\underline{\boldsymbol{\sigma}}_{h}, \underline{\boldsymbol{\tau}}_{h}\right)_{T}=\left(\nabla \mathbf{u}_{h}-p_{h} \underline{\boldsymbol{I}}, \underline{\boldsymbol{\tau}}_{h}\right)_{T}-\theta \sum_{F \in \mathcal{F}_{T}}\left\langle\omega_{F} \underline{\boldsymbol{\tau}}_{h} \mathbf{n}_{F}, \llbracket \mathbf{u}_{h} \rrbracket\right\rangle_{F},
$$

where $\omega_{F}:=\frac{1}{2}$ for $F \in \partial \mathcal{T}_{h}^{\text {int }}$ and $\omega_{F}:=1$ for $F \in \partial \mathcal{T}_{h}^{\text {ext }}$. Observe that the quantities prescribing the moments of $\underline{\boldsymbol{\sigma}}_{h} \mathbf{n}_{F}$ are uniquely defined for each side $F \in \partial \mathcal{T}_{h}$, whence the continuity of the normal trace of $\underline{\boldsymbol{\sigma}}_{h}$. The two following lemmas are of decisive importance, implying (4.3) and (6.2), respectively.

Lemma 7.1 (Reconstructed flux in the discontinuous Galerkin method) For $T \in \mathcal{T}_{h}$, let $\underline{\boldsymbol{\sigma}}_{h}$ be defined by (7.6)-(7.7). Then, there holds

$$
\left(\nabla \cdot \underline{\boldsymbol{\sigma}}_{h}+\mathbf{f}, \mathbf{v}_{h}\right)_{T}=0 \quad \forall \mathbf{v}_{h} \in\left[\mathbb{P}_{l}(T)\right]^{d},
$$

i.e.,

$$
\left.\left(\nabla \cdot \underline{\boldsymbol{\sigma}}_{h}\right)\right|_{T}=-\left.\left(\boldsymbol{\Pi}_{l} \mathbf{f}\right)\right|_{T}
$$

where $\boldsymbol{\Pi}_{l}$ is the $\mathbf{L}^{2}$-orthogonal projection onto $\left[\mathbb{P}_{l}\left(\mathcal{T}_{h}\right)\right]^{d}$. Thus, in particular, (4.3) holds.

Proof Let $T \in \mathcal{T}_{h}$ and let $\mathbf{v}_{h} \in\left[\mathbb{P}_{l}(T)\right]^{d}$. Owing to the Green theorem, it holds

$$
\left(\nabla \cdot \underline{\boldsymbol{\sigma}}_{h}, \mathbf{v}_{h}\right)_{T}=-\left(\underline{\boldsymbol{\sigma}}_{h}, \nabla \mathbf{v}_{h}\right)_{T}+\sum_{F \in \mathcal{F}_{T}}\left\langle\underline{\boldsymbol{\sigma}}_{h} \mathbf{n}_{T}, \mathbf{v}_{h}\right\rangle_{F}=: T_{1}+T_{2} .
$$

Since $\left.\nabla \mathbf{v}_{h}\right|_{T} \in\left[\mathbb{P}_{l-1}(T)\right]^{d \times d}$, using (7.7) yields

$$
T_{1}=-\left(\nabla \mathbf{u}_{h}-p_{h} \underline{\boldsymbol{I}}, \nabla \mathbf{v}_{h}\right)_{T}+\theta \sum_{F \in \mathcal{F}_{T}}\left\langle\omega_{F} \nabla \mathbf{v}_{h} \mathbf{n}_{F}, \llbracket \mathbf{u}_{h} \rrbracket\right\rangle_{F} .
$$

Furthermore, the fact that $\left.\mathbf{v}_{h}\right|_{F} \in\left[\mathbb{P}_{l}(F)\right]^{d}$ for all $F \in \mathcal{F}_{T}$ and (7.6) yield

$$
T_{2}=\sum_{F \in \mathcal{F}_{T}}\left\langle\left\{\nabla \mathbf{u}_{h}-p_{h} \underline{\boldsymbol{I}}\right\} \mathbf{n}_{F}-\gamma_{F} h_{F}^{-1} \llbracket \mathbf{u}_{h} \rrbracket, \mathbf{n}_{T} \cdot \mathbf{n}_{F} \mathbf{v}_{h}\right\rangle_{F} .
$$

Extend $\mathbf{v}_{h}$ by $\mathbf{0}$ outside of $T$. Using the above identities, (7.2), (7.3), and (7.4a) yields

$$
T_{1}+T_{2}=-a_{h}\left(\mathbf{u}_{h}, \mathbf{v}_{h}\right)-b_{h}\left(\mathbf{v}_{h}, p_{h}\right)=-\left(\mathbf{f}, \mathbf{v}_{h}\right)_{T},
$$

whence (7.8) is valid. Finally, (7.9) results from (7.8) and the fact that $\left.\nabla \cdot \underline{\boldsymbol{\sigma}}_{h}\right|_{T} \in\left[\mathbb{P}_{l}(T)\right]^{d}$.

Lemma 7.2 (Approximation property of the reconstructed flux in the discontinuous Galerkin method) For $T \in \mathcal{T}_{h}$, let $\underline{\boldsymbol{\sigma}}_{h}$ be defined by (7.6)-(7.7). Then (6.2) holds. 
Proof The proof follows the lines of that in the case of second-order elliptic equations. Recall that in the present case $\left(\mathcal{S}_{h}=\mathcal{T}_{h}\right), \mathcal{T}_{h}$ is shape-regular by Assumption 6.1. Using the equivalence of norms on finite-dimensional spaces, the Piola transformation, and scaling arguments, one shows that for all $T \in \mathcal{T}_{h}$ and all $\underline{\boldsymbol{v}}_{h} \in \underline{\boldsymbol{\Sigma}}^{l}(T)$

$$
\left\|\underline{\boldsymbol{v}}_{h}\right\|_{T}^{2} \lesssim\left\{h_{T} \sum_{F \in \mathcal{F}_{T}}\left\|\underline{\boldsymbol{v}}_{h} \mathbf{n}_{F}\right\|_{F}^{2}+\left(\sup _{\underline{\boldsymbol{\tau}}_{h} \in\left[\mathbb{P}_{l-1}(T)\right]^{d \times d}} \frac{\left(\underline{\boldsymbol{v}}_{h}, \underline{\boldsymbol{\tau}}_{h}\right)_{T}}{\left\|\underline{\boldsymbol{\tau}}_{h}\right\|_{T}}\right)^{2}\right\} .
$$

Define $\underline{\boldsymbol{v}}_{h}:=\nabla \mathbf{u}_{h}-p_{h} \underline{\boldsymbol{I}}-\underline{\boldsymbol{\sigma}}_{h}$. Then, using (7.7) and the Cauchy-Schwarz and inverse inequalities, we get

$$
\left(\underline{\boldsymbol{v}}_{h}, \underline{\boldsymbol{\tau}}_{h}\right)_{T}=\theta \sum_{F \in \mathcal{F}_{T}}\left\langle\omega_{F} \underline{\boldsymbol{\tau}}_{h} \mathbf{n}_{F}, \llbracket \mathbf{u}_{h} \rrbracket\right\rangle_{F} \lesssim|\theta| h_{T}^{-1 / 2}\left\|\underline{\boldsymbol{\tau}}_{h}\right\|_{T} \sum_{F \in \mathcal{F}_{T}}\left\|\llbracket \mathbf{u}_{h} \rrbracket\right\|_{F} .
$$

Note that (7.6) gives

$$
\left.\underline{\boldsymbol{\sigma}}_{h} \mathbf{n}_{F}\right|_{F}=\left\{\left\{\nabla \mathbf{u}_{h}-p_{h} \underline{\boldsymbol{I}}\right\}\right\} \mathbf{n}_{F}-\gamma_{F} h_{F}^{-1} \boldsymbol{\Pi}_{l}\left(\llbracket \mathbf{u}_{h} \rrbracket\right) .
$$

Thus, using (7.10) and the above developments, we have

$$
\begin{aligned}
\left\|\underline{\boldsymbol{v}}_{h}\right\|_{T}^{2} \lesssim & \left\{h_{T} \sum_{F \in \mathcal{F}_{T}^{\text {int }}}\left\|\llbracket \nabla \mathbf{u}_{h}-p_{h} \underline{\boldsymbol{I}} \rrbracket \mathbf{n}_{F}\right\|_{F}^{2}+h_{T} \sum_{F \in \mathcal{F}_{T}}\left\|\gamma_{F} h_{F}^{-1} \boldsymbol{\Pi}_{l}\left(\llbracket \mathbf{u}_{h} \rrbracket\right)\right\|_{F}^{2}\right. \\
& \left.+|\theta|^{2} h_{T}^{-1} \sum_{F \in \mathcal{F}_{T}}\left\|\llbracket \mathbf{u}_{h} \rrbracket\right\|_{F}^{2}\right\},
\end{aligned}
$$

whence (6.2) follows.

\subsection{Conforming and conforming stabilized methods}

We will show here how locally efficient a posteriori error estimates can be obtained for conforming and conforming stabilized methods using Corollary 5.1 and Theorem 6.1. We suppose that $\mathcal{T}_{h}$ consists of simplices and is matching. In this section, $\mathbf{V}_{h} \subset \mathbf{V}$, so that we systematically set $\mathbf{s}_{h}=\mathbf{u}_{h}$ throughout this section.

The conforming methods for the problem (2.3a)-(2.3b) that we consider read: find $\left(\mathbf{u}_{h}, p_{h}\right) \in \mathbf{V}_{h} \times Q_{h}$ such that

$$
\begin{aligned}
a\left(\mathbf{u}_{h}, \mathbf{v}_{h}\right)+b\left(\mathbf{v}_{h}, p_{h}\right) & =\left(\mathbf{f}, \mathbf{v}_{h}\right) & & \forall \mathbf{v}_{h} \in \mathbf{V}_{h}, \\
b\left(\mathbf{u}_{h}, q_{h}\right) & =0 & & \forall q_{h} \in Q_{h} .
\end{aligned}
$$

In particular, we consider the Taylor-Hood family [54,16], where, for $k \geq 1$,

$$
\mathbf{V}_{h}=\left[\mathbb{P}_{k+1}\left(\mathcal{T}_{h}\right)\right]^{d} \cap \mathbf{V}, \quad Q_{h}=\mathbb{P}_{k}\left(\mathcal{T}_{h}\right) \cap C(\Omega) \cap Q .
$$

The Mini element [7], where

$$
\mathbf{V}_{h}:=\left[\mathbb{P}_{1}^{\mathrm{b}}\left(\mathcal{T}_{h}\right)\right]^{d} \cap \mathbf{V}, \quad Q_{h}=\mathbb{P}_{1}\left(\mathcal{T}_{h}\right) \cap C(\Omega) \cap Q,
$$

where $\mathbb{P}_{1}^{\mathrm{b}}\left(\mathcal{T}_{h}\right)$ stands for $\mathbb{P}_{1}\left(\mathcal{T}_{h}\right)$ enriched by bubbles, is likewise considered. We also include the lowest-order methods, namely the cross-grid $\mathbb{P}_{1}-\mathbb{P}_{1}$ element [48], where

$$
\mathbf{V}_{h}:=\left[\mathbb{P}_{1}\left(\mathcal{T}_{h}^{\mathrm{c}}\right)\right]^{d} \cap \mathbf{V}, \quad Q_{h}=\mathbb{P}_{1}\left(\mathcal{T}_{h}\right) \cap C(\Omega) \cap Q,
$$


with $\mathcal{T}_{h}^{\mathrm{c}}$ formed from $\mathcal{T}_{h}$ by adding the barycenter as a node and cutting the simplex into $(d+1)$ subsimplices, and the $\mathbb{P}_{1}$ iso $\mathbb{P}_{2}-\mathbb{P}_{1}$ element [12], where

$$
\mathbf{V}_{h}:=\left[\mathbb{P}_{1}\left(\mathcal{T}_{h / 2}\right)\right]^{d} \cap \mathbf{V}, \quad Q_{h}=\mathbb{P}_{1}\left(\mathcal{T}_{h}\right) \cap C(\Omega) \cap Q,
$$

with $\mathcal{T}_{h / 2}$ formed from $\mathcal{T}_{h}$ by adding the edge barycenters as nodes and cutting the simplex into $2^{d}$ subsimplices.

We also consider the conforming stabilized methods written in the general form: find $\left(\mathbf{u}_{h}, p_{h}\right) \in \mathbf{V}_{h} \times Q_{h}$ such that

$$
\begin{aligned}
a\left(\mathbf{u}_{h}, \mathbf{v}_{h}\right)+b\left(\mathbf{v}_{h}, p_{h}\right)+t_{h}\left(\mathbf{u}_{h}, p_{h} ; \mathbf{v}_{h}\right) & =\left(\mathbf{f}, \mathbf{v}_{h}\right) & & \forall \mathbf{v}_{h} \in \mathbf{V}_{h}, \\
s_{h}\left(\mathbf{u}_{h}, p_{h} ; q_{h}\right)+b\left(\mathbf{u}_{h}, q_{h}\right) & =0 & & \forall q_{h} \in Q_{h} .
\end{aligned}
$$

Let $\delta>0$ be a parameter and let

$$
\mathbf{V}_{h}:=\left[\mathbb{P}_{k}\left(\mathcal{T}_{h}\right)\right]^{d} \cap \mathbf{V}, \quad Q_{h}=\mathbb{P}_{k}\left(\mathcal{T}_{h}\right) \cap C(\Omega) \cap Q .
$$

Then the Brezzi-Pitkäranta method [18] uses

$$
\begin{aligned}
& s_{h}\left(\mathbf{u}_{h}, p_{h} ; q_{h}\right)=-\delta \sum_{T \in \mathcal{T}_{h}} h_{T}^{2}\left(\nabla p_{h}, \nabla q_{h}\right)_{T} \\
& t_{h}\left(\mathbf{u}_{h}, p_{h} ; \mathbf{v}_{h}\right)=0
\end{aligned}
$$

and $k=1$, the Hughes-Franca-Balestra method [41] uses

$$
\begin{aligned}
& s_{h}\left(\mathbf{u}_{h}, p_{h} ; q_{h}\right)=\delta \sum_{T \in \mathcal{T}_{h}} h_{T}^{2}\left(\mathbf{f}+\Delta \mathbf{u}_{h}-\nabla p_{h}, \nabla q_{h}\right)_{T} \\
& t_{h}\left(\mathbf{u}_{h}, p_{h} ; \mathbf{v}_{h}\right)=0
\end{aligned}
$$

and $k \geq 1$, and the Brezzi-Douglas method [15] uses

$$
\begin{aligned}
& s_{h}\left(\mathbf{u}_{h}, p_{h} ; q_{h}\right)=\delta \sum_{T \in \mathcal{T}_{h}} h_{T}^{2}\left\{\left(\mathbf{f}-\nabla p_{h}, \nabla q_{h}\right)_{T}+\left\langle\Delta \mathbf{u}_{h} \cdot \mathbf{n}_{T}, q_{h}\right\rangle_{\partial T \cap \partial \Omega}\right\} \\
& t_{h}\left(\mathbf{u}_{h}, p_{h} ; \mathbf{v}_{h}\right)=0
\end{aligned}
$$

and $k \geq 1$.

For

$$
\mathbf{V}_{h}:=\left[\mathbb{P}_{k}\left(\mathcal{T}_{h}\right)\right]^{d} \cap \mathbf{V}, \quad Q_{h}=\mathbb{P}_{k-1}\left(\mathcal{T}_{h}\right) \cap C(\Omega) \cap Q,
$$

$k \geq 2$, and a parameter $\rho=-1,1$, we also consider the following optimally converging Hughes-Franca-Balestra family [41], see [36,35] for an error analysis:

$$
\begin{aligned}
& s_{h}\left(\mathbf{u}_{h}, p_{h} ; q_{h}\right)=\delta \sum_{T \in \mathcal{T}_{h}} h_{T}^{2}\left(\mathbf{f}+\Delta \mathbf{u}_{h}-\nabla p_{h}, \nabla q_{h}\right)_{T} \\
& t_{h}\left(\mathbf{u}_{h}, p_{h} ; \mathbf{v}_{h}\right)=\delta \rho \sum_{T \in \mathcal{T}_{h}} h_{T}^{2}\left(\mathbf{f}+\Delta \mathbf{u}_{h}-\nabla p_{h}, \Delta \mathbf{v}_{h}\right)_{T} .
\end{aligned}
$$




\subsubsection{Lowest-order continuous pressure elements}

We consider here the lowest-order methods with the velocity and pressure spaces formed by continuous piecewise $\mathbb{P}_{1}$ polynomials, namely the cross-grid $\mathbb{P}_{1}-\mathbb{P}_{1}$ element, the $\mathbb{P}_{1}$ iso $\mathbb{P}_{2}-\mathbb{P}_{1}$ element, and the above stabilized methods with $k=1$. In the sequel, for the first two methods, $\mathcal{T}_{h}^{\mathrm{c}}$ or $\mathcal{T}_{h / 2}$ is to be substituted systematically in place of $\mathcal{T}_{h}$. We follow the approach introduced in $[45,60,63]$.

First, we need to introduce some more notation. Let the dual mesh $\mathcal{D}_{h}$ be formed around each vertex of $\mathcal{T}_{h}$ using the edge, elements, (and face in 3D) barycenters as indicated in the left part of Figure 7.1. Let $\mathcal{D}_{h}^{\text {int }}$ correspond to the interior vertices and $\mathcal{D}_{h}^{\text {ext }}$ to the boundary ones. Finally, we cut each $D \in$ $\mathcal{D}_{h}$ into a simplicial mesh $\mathcal{S}_{D}$ as indicated in the right part of Figure 7.1; the matching simplicial submesh $\mathcal{S}_{h}$ of $\mathcal{T}_{h}$ (and of $\mathcal{D}_{h}$ ), needed in Assumption 6.1, is created by collecting the local meshes $\mathcal{S}_{D}$. We denote by $\mathcal{F}_{D}$ all the sides of a given $D \in \mathcal{D}_{h}$, by $\partial \mathcal{S}_{h}$ all the sides of $\mathcal{S}_{h}$, and by $\partial \mathcal{S}_{h}^{\text {int }}$ all the interior sides of $\mathcal{S}_{h}$. Similarly, for $D \in \mathcal{D}_{h}$, we will employ the notation $\partial \mathcal{S}_{D}$ for all the sides of $\mathcal{S}_{D}, \partial \mathcal{S}_{D}^{\text {int }}$ for all the interior sides of $\mathcal{S}_{D}$, and $\partial \mathcal{S}_{D}^{\text {ext }}$ for all the boundary sides of $\mathcal{S}_{D}$. The notation introduced in Section 3.1 for the mesh $\mathcal{T}_{h}$ will be used in this section also for the meshes $\mathcal{D}_{h}$ and $\mathcal{S}_{h}$. For a vertex $V \in \mathcal{V}_{h}$, let $\psi_{V}$ be the associated $\mathbb{P}_{1}$ finite element "hat" basis function. Let $\boldsymbol{\psi}_{V, i}, i=1, \ldots, d$, be its vector variants such that $\boldsymbol{\psi}_{V, i}^{i}=\psi_{V}, \boldsymbol{\psi}_{V, i}^{j}=0$ for $j=1, \ldots, d, j \neq i$.

For a side $F \in \partial \mathcal{S}_{h}^{\text {int }}$ such that $F \subset \partial D$ for some $D \in \mathcal{D}_{h}$, define the normal flux functions

$$
\boldsymbol{\Upsilon}_{F}\left(\mathbf{u}_{h}\right):=\left.\left(\nabla \mathbf{u}_{h} \mathbf{n}_{F}\right)\right|_{F} .
$$

Note that all such sides lie inside some $T \in \mathcal{T}_{h}$, cf. Figure 7.1 , so that $\nabla \mathbf{u}_{h}$ is indeed univalued thereon. The following important property holds for all the above-listed methods.

Lemma 7.3 (Local conservativity of lowest-order conforming methods) Let $\mathbf{f}$ be piecewise constant on $\mathcal{T}_{h}$ and let $\left(\mathbf{u}_{h}, p_{h}\right) \in \mathbf{V}_{h} \times Q_{h}$ be given by (7.11a)-(7.11b) or by (7.12a)-(7.12b) for any of the spaces described above. Let $\boldsymbol{\Upsilon}_{F}\left(\mathbf{u}_{h}\right)$ be given by (7.14). Then

$$
\begin{array}{r}
\sum_{F \in \mathcal{F}_{D}}\left\langle\boldsymbol{\Upsilon}_{F}\left(\mathbf{u}_{h}\right) \mathbf{n}_{D} \cdot \mathbf{n}_{F}, \mathbf{e}_{i}\right\rangle_{F}-\left(\nabla p_{h}, \mathbf{e}_{i}\right)_{D}+\left(\mathbf{f}, \mathbf{e}_{i}\right)_{D}=0, \\
i=1, \ldots, d \quad \forall D \in \mathcal{D}_{h}^{\text {int }} .
\end{array}
$$

Proof For a given dual volume $D \in \mathcal{D}_{h}^{\text {int }}$ and associated vertex $V$, fix $i \in$ $\{1, \ldots, d\}$ and consider $\boldsymbol{\psi}_{V, i}$ as the test function $\mathbf{v}_{h}$ in (7.11a) or (7.12a). Recall that the support of $\boldsymbol{\psi}_{V, i}$ is given by $\mathcal{T}_{V}$, all the elements of $\mathcal{T}_{h}$ sharing $V$. Then, under the assumption that $\mathbf{f}$ is piecewise constant on $\mathcal{T}_{h}$,

$$
\left(\mathbf{f}, \boldsymbol{\psi}_{V, i}\right)_{\mathfrak{T}_{V}}=\left(\mathbf{f}, \mathbf{e}_{i}\right)_{D}
$$

easily follows as $|D \cap T|=|T| /(d+1)$ for all $T \in \mathfrak{T}_{V}$, (cf., e.g., [63, Lemma 3.11]). Next, one derives

$$
\left(\nabla \mathbf{u}_{h}, \nabla \boldsymbol{\psi}_{V, i}\right)_{\mathfrak{T}_{V}}=-\left\langle\nabla \mathbf{u}_{h} \mathbf{n}_{D}, \mathbf{e}_{i}\right\rangle_{\partial D}
$$

as in [8, Lemma 3] or [63, Lemma 3.8]. Thus, using (7.14),

$$
\left(\nabla \mathbf{u}_{h}, \nabla \boldsymbol{\psi}_{V, i}\right)_{\mathfrak{T}_{V}}=-\sum_{F \in \mathcal{F}_{D}}\left\langle\boldsymbol{\Upsilon}_{F}\left(\mathbf{u}_{h}\right) \mathbf{n}_{D} \cdot \mathbf{n}_{F}, \mathbf{e}_{i}\right\rangle_{F}
$$



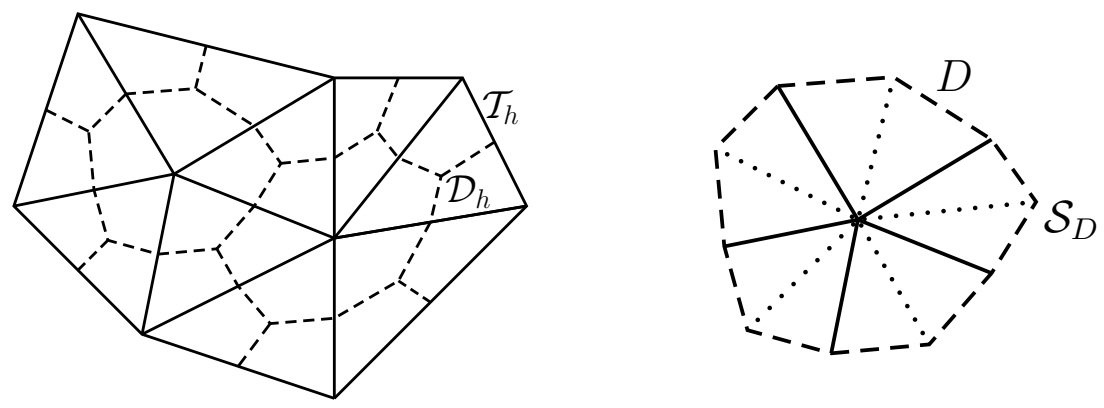

Fig. 7.1 Dual mesh $\mathcal{D}_{h}$ (left) and a simplicial submesh $\mathcal{S}_{D}$ of $D \in \mathcal{D}_{h}$ (right) for conforming methods in two space dimensions

Next, using the assumption $p_{h} \in \mathbb{P}_{1}\left(\mathcal{T}_{h}\right) \cap C(\Omega)$, implying $p_{h} \in H^{1}(\Omega)$, the Green theorem, and the fact that $\boldsymbol{\psi}_{V, i}=\mathbf{0}$ on $\partial \mathfrak{T}_{V}$, one comes to

$$
b\left(\boldsymbol{\psi}_{V, i}, p_{h}\right)=-\left(\nabla \cdot \boldsymbol{\psi}_{V, i}, p_{h}\right)_{\mathfrak{T}_{V}}=\left(\boldsymbol{\psi}_{V, i}, \nabla p_{h}\right)_{\mathfrak{T}_{V}} .
$$

The above right-hand side can still be rewritten equivalently as

$$
\left(\boldsymbol{\psi}_{V, i}, \nabla p_{h}\right)_{\mathfrak{T}_{V}}=\left(\mathbf{e}_{i}, \nabla p_{h}\right)_{D}
$$

This follows from the fact that $\nabla p_{h}$ is piecewise constant on $\mathcal{T}_{h}$, so we can use the same arguments as for obtaining (7.16). Thus, combining the above arguments, (7.15) is implied by (7.11a) or by (7.12a).

Remark 7.1 (Lemma 7.3) Note that, actually, only (7.11a) or (7.12a), neither (7.11b) nor (7.12b), is needed in Lemma 7.3.

We will now define a suitable $\underline{\boldsymbol{\sigma}}_{h} \in \underline{\boldsymbol{H}}(\operatorname{div}, \Omega)$; more precisely, we will construct $\underline{\boldsymbol{\sigma}}_{h}$ in the Raviart-Thomas-Nédélec space $\underline{\boldsymbol{\Sigma}}^{0}\left(\mathcal{S}_{h}\right)$, see $(7.5)$, on the fine simplicial mesh $\mathcal{S}_{h}$. Prior to proceeding to a construction ensuring (5.9) (that is, a local conservation property on the mesh $\mathcal{T}_{h}$ ), let us make the following remark.

Remark 7.2 (Simple construction of $\underline{\boldsymbol{\sigma}}_{h}$ ) Following $[60,63]$, the simplest construction of $\underline{\boldsymbol{\sigma}}_{h} \in \underline{\boldsymbol{\Sigma}}^{0}\left(\mathcal{S}_{h}\right)$ is by

$$
\underline{\boldsymbol{\sigma}}_{h} \mathbf{n}_{F}:=\left\{\left\{\nabla \mathbf{u}_{h} \mathbf{n}_{F}\right\} \quad \forall F \in \partial \mathcal{S}_{h},\right.
$$

that is, we merely prescribe the degrees of freedom of $\underline{\boldsymbol{\sigma}}_{h}$ by averaging the normal components of the discontinuous approximate flux $\nabla \mathbf{u}_{h}$ over those sides of the mesh $\mathcal{S}_{h}$ which are contained in $\partial \mathcal{T}_{h}$ and by setting directly $\nabla \mathbf{u}_{h} \mathbf{n}_{F}$ on those sides of the mesh $\mathcal{S}_{h}$ which are not contained in $\partial \mathcal{T}_{h}$. The flux $\underline{\boldsymbol{\sigma}}_{h}$ defined by (7.18) (which is consistent with (7.14)) in virtue of (7.15) clearly satisfies (5.9), but on the mesh $\mathcal{D}_{h}^{\text {int }}$ and not on the mesh $\mathcal{T}_{h}$. The upper bound would then needed to be written on the mesh $\mathcal{D}_{h}$ instead of $\mathcal{T}_{h}$, following $[60,63]$. The proof of the approximation property (6.3) is in this case straightforward: using (7.18) and (7.10), on $T \in \mathcal{S}_{h}$,

$$
\left\|\nabla \mathbf{u}_{h}-\underline{\boldsymbol{\sigma}}_{h}\right\|_{T} \lesssim\left\{h_{F} \sum_{F \in \mathcal{F}_{T}}\left\|\llbracket \nabla \mathbf{u}_{h} \rrbracket \mathbf{n}_{F}\right\|_{F}^{2}\right\}^{1 / 2}
$$

whence (6.3) follows taking into account the fact that $\llbracket p_{h} \underline{\boldsymbol{I n}_{F}} \rrbracket$ is zero since $p_{h} \in C(\Omega)$. 
Let us now define $\underline{\boldsymbol{\sigma}}_{h} \in \underline{\boldsymbol{\Sigma}}^{0}\left(\mathcal{S}_{h}\right)$ such that (5.9) holds, that is, such that the local conservation property is satisfied on the original mesh $\mathcal{T}_{h}$. For this purpose, we adapt to the present setting the approach of $[33,63]$. It consists in mixed finite element solutions of local Neumann/Dirichlet problems. A local linear system on each $D \in \mathcal{D}_{h}$ has to be solved here but numerical experiments reveal better performance of this approach.

Let a dual volume $D \in \mathcal{D}_{h}$ and a polynomial degree $l \geq 0$ be given. In this section, $l=0$, but $l \geq 1$ will be required later for higher-order conforming methods. Let the normal flux function $\boldsymbol{\Upsilon}_{F}\left(\mathbf{u}_{h}\right)$ be defined by (7.14). We generalize this notation to $\boldsymbol{\Upsilon}_{F}\left(\mathbf{u}_{h}, p_{h}\right)$, required once again later for higherorder conforming methods. Denote

$$
\underline{\boldsymbol{\Sigma}}_{\mathrm{N}}^{l}\left(\mathcal{S}_{D}\right):=\left\{\underline{\boldsymbol{v}}_{h} \in \underline{\boldsymbol{\Sigma}}^{l}\left(\mathcal{S}_{D}\right) ; \underline{\boldsymbol{v}}_{h} \mathbf{n}_{F}=\boldsymbol{\Upsilon}_{F}\left(\mathbf{u}_{h}, p_{h}\right) \quad \forall F \in \partial \mathcal{S}_{h}^{\text {int }}, F \subset \partial D\right\} .
$$

This is a space of Raviart-Thomas-Nédélec tensor stresses on the given dual volume $D$ whose normal fluxes are given by $\boldsymbol{\Upsilon}_{F}\left(\mathbf{u}_{h}, p_{h}\right)$ on $\partial D \backslash \partial \Omega$. Let $\boldsymbol{\Pi}_{l}$ denote the $\mathbf{L}^{2}$-orthogonal projection onto $\left[\mathbb{P}_{l}\left(\mathcal{S}_{h}\right)\right]^{d}$. We then define $\underline{\boldsymbol{\sigma}}_{h}$, the equilibrated flux being at the heart of our analysis, in the space $\underline{\boldsymbol{\Sigma}}^{l}\left(\mathcal{S}_{h}\right)$ by solving on each $D \in \mathcal{D}_{h}$ the following minimization problem:

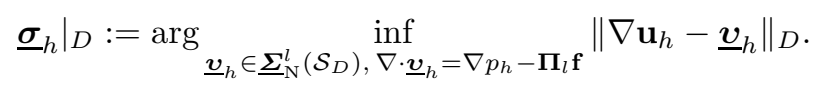

Note that the fact that $\boldsymbol{\Upsilon}_{F}\left(\mathbf{u}_{h}, p_{h}\right)$ are univalued and the definition (7.19) ensure that such a flux $\underline{\boldsymbol{\sigma}}_{h}$ has the normal component continuous and thus indeed belongs to $\underline{\boldsymbol{\Sigma}}^{l}\left(\mathcal{S}_{h}\right)$.

Let $\underline{\boldsymbol{\Sigma}}_{\mathrm{N}, 0}^{l}\left(\mathcal{S}_{D}\right)$ be as $\underline{\boldsymbol{\Sigma}}_{\mathrm{N}}^{l}\left(\mathcal{S}_{D}\right)$ but with the normal flux condition $\underline{\boldsymbol{v}}_{h} \mathbf{n}_{F}=$ $\mathbf{0}$ on $F \in \partial \mathcal{S}_{h}^{\text {int }}, F \subset \partial D$. Let $\left[\mathbb{P}_{l}^{*}\left(\mathcal{S}_{D}\right)\right]^{d}$ be spanned by piecewise constant vectors on $\mathcal{S}_{D}$ with zero mean on $D$ in each component when $D \in \mathcal{D}_{h}^{\text {int }}$; when $D \in \mathcal{D}_{h}^{\text {ext }}$, the mean value condition is not imposed. Then it is easy to show, cf. [33], that (7.20) is equivalent to finding $\underline{\boldsymbol{\sigma}}_{h} \in \underline{\boldsymbol{\Sigma}}_{\mathrm{N}}^{l}\left(\mathcal{S}_{D}\right)$ and $\mathbf{r}_{h} \in\left[\mathbb{P}_{l}^{*}\left(\mathcal{S}_{D}\right)\right]^{d}$ such that

$$
\begin{aligned}
\left(\underline{\boldsymbol{\sigma}}_{h}-\nabla \mathbf{u}_{h}, \underline{\boldsymbol{v}}_{h}\right)_{D}+\left(\mathbf{r}_{h}, \nabla \cdot \underline{\boldsymbol{v}}_{h}\right)_{D}=0 & \forall \underline{\boldsymbol{v}}_{h} \in \underline{\boldsymbol{\Sigma}}_{\mathrm{N}, 0}^{l}\left(\mathcal{S}_{D}\right), \\
-\left(\nabla \cdot \underline{\boldsymbol{\sigma}}_{h}, \boldsymbol{\phi}_{h}\right)_{D}-\left(\mathbf{f}-\nabla p_{h}, \boldsymbol{\phi}_{h}\right)_{D}=0 & \forall \boldsymbol{\phi}_{h} \in\left[\mathbb{P}_{l}^{*}\left(\mathcal{S}_{D}\right)\right]^{d} .
\end{aligned}
$$

The existence and uniqueness of a solution to the above system are standard. This system is a mixed finite element approximation of a local Neumann problem on $D \in \mathcal{D}_{h}^{\text {int }}$; the Neumann boundary conditions are given by the normal flux functions $\boldsymbol{\Upsilon}_{F}\left(\mathbf{u}_{h}, p_{h}\right)$. Note in particular that $\boldsymbol{\Upsilon}_{F}\left(\mathbf{u}_{h}, p_{h}\right)$ satisfy the Neumann compatibility condition by (7.15). When $D \in \mathcal{D}_{h}^{\text {ext }}$, this system is a mixed finite element approximation of a local Neumann/Dirichlet problem; homogeneous Dirichlet boundary condition is prescribed on $\partial D \cap \partial \Omega$.

These developments imply:

Lemma 7.4 (Reconstructed flux in lowest-order conforming methods) Let $\mathbf{f}$ be piecewise constant on $\mathcal{T}_{h}$ and let $\left(\mathbf{u}_{h}, p_{h}\right) \in \mathbf{V}_{h} \times Q_{h}$ be given by (7.11a)-(7.11b) or by (7.12a)-(7.12b) for any of the lowest-order methods. Let $\boldsymbol{\Upsilon}_{F}\left(\mathbf{u}_{h}\right)$ be given by (7.14) and prescribe $\underline{\boldsymbol{\sigma}}_{h}$ by $(7.20)$, with $l=0$. Then (5.9) holds. More precisely,

$$
\left.\left(\nabla \cdot \underline{\boldsymbol{\sigma}}_{h}\right)\right|_{T}=\left.\left(\nabla p_{h}-\mathbf{f}\right)\right|_{T} \quad \forall T \in \mathcal{S}_{h} .
$$

To finish this section, we have: 
Lemma 7.5 (Approximation property of the reconstructed flux in lowest-order conforming methods) Let the assumptions of Lemma 7.4 be verified. Then the approximation property (6.3) holds.

Proof Let $D \in \mathcal{D}_{h}$ and let $\underline{\boldsymbol{\sigma}}_{h} \in \underline{\boldsymbol{\Sigma}}_{\mathrm{N}}^{0}\left(\mathcal{S}_{D}\right)$ and $\mathbf{r}_{h} \in\left[\mathbb{P}_{0}^{*}\left(\mathcal{S}_{D}\right)\right]^{d}$ be given by (7.21a)-(7.21b). Extending the approach of [59, Section 4.1] (cf. also [6, 5]) to the vector case, we define a postprocessing $\tilde{\mathbf{r}}_{h}$ of $\mathbf{r}_{h}$ such that

$$
\begin{aligned}
\left.\nabla \tilde{\mathbf{r}}_{h}\right|_{T} & =\left.\left(\underline{\boldsymbol{\sigma}}_{h}-\nabla \mathbf{u}_{h}\right)\right|_{T} & & \forall T \in \mathcal{S}_{D}, \\
\frac{\left(\tilde{\mathbf{r}}_{h}, \mathbf{e}_{i}\right)_{T}}{|T|} & =\left.\mathbf{r}_{h}^{i}\right|_{T}, & i & =1, \ldots, d, \quad \forall T \in \mathcal{S}_{D} .
\end{aligned}
$$

Note that this is a cheap local procedure. It follows from (7.23a), (7.23b), and (7.21a) that

$$
\left(\nabla \tilde{\mathbf{r}}_{h}, \underline{\boldsymbol{v}}_{h}\right)_{D}+\left(\tilde{\mathbf{r}}_{h}, \nabla \cdot \underline{\boldsymbol{v}}_{h}\right)_{D}=0 \quad \forall \underline{\boldsymbol{v}}_{h} \in \underline{\boldsymbol{\Sigma}}_{\mathrm{N}, 0}^{l}\left(\mathcal{S}_{D}\right) .
$$

Fixing one $F \in \partial \mathcal{S}_{D}^{\text {int }}$, choosing the basis functions of $\underline{\boldsymbol{\Sigma}}_{\mathrm{N}, 0}^{l}\left(\mathcal{S}_{D}\right)$ having nonzero normal trace only across this side, and using the Green theorem, we arrive at

$$
\left\langle\llbracket \tilde{\mathbf{r}}_{h} \rrbracket, \mathbf{e}_{i}\right\rangle_{F}=0, \quad i=1, \ldots, d .
$$

This means that the postprocessed $\tilde{\mathbf{r}}_{h}$ has the mean value of the jump in each component equal to zero on the interior sides of $\mathcal{S}_{D}$. Alternatively, we can say that $\tilde{\mathbf{r}}_{h}$ has means of traces continuous on the interior sides of $\mathcal{S}_{D}$.

If $D \in \mathcal{D}_{h}^{\text {ext }}$, we arrive similarly at

$$
\left\langle\tilde{\mathbf{r}}_{h}, \mathbf{e}_{i}\right\rangle_{F}=0, \quad i=1, \ldots, d,
$$

for all $F \in \partial \mathcal{S}_{D}^{\text {ext }}$ such that $F \subset \partial \Omega$. Thus, on exterior sides of $\mathcal{S}_{D}$ belonging to $\partial \Omega$, the mean value of each component of $\tilde{\mathbf{r}}_{h}$ is zero.

Finally, for $D \in \mathcal{D}_{h}^{\text {int }}$, we have that $\left(\mathbf{r}_{h}, \mathbf{e}_{i}\right)_{D}=0, i=1, \ldots, d$, from the definition of $\left[\mathbb{P}_{0}^{*}\left(\mathcal{S}_{D}\right)\right]^{d}$. From this fact and $(7.23 \mathrm{~b})$, we deduce that

$$
\left(\tilde{\mathbf{r}}_{h}, \mathbf{e}_{i}\right)_{D}=0, \quad i=1, \ldots, d .
$$

Thus, on dual volumes not touching the boundary, the mean value of each component of $\tilde{\mathbf{r}}_{h}$ is zero.

We denote by $\mathbf{M}\left(\mathcal{S}_{D}\right) \subset\left[\mathbb{P}_{2}\left(\mathcal{S}_{D}\right)\right]^{d}$ the corresponding space of polynomials verifying (7.24), (7.25), and (7.26). Using the above developments, we have

$$
\left\|\nabla \mathbf{u}_{h}-\underline{\boldsymbol{\sigma}}_{h}\right\|_{D}=\sup _{\mathbf{m}_{h} \in \mathbf{M}\left(\mathcal{S}_{D}\right),\left\|\nabla \mathbf{m}_{h}\right\|_{D}=1}\left(\nabla \mathbf{u}_{h}-\underline{\boldsymbol{\sigma}}_{h}, \nabla \mathbf{m}_{h}\right)_{D}
$$

We now develop the right-hand side of (7.27). Using the Green theorem, the fact that $\nabla \cdot \underline{\boldsymbol{\sigma}}_{h}=\nabla p_{h}-\mathbf{f}$ for all $T \in \mathcal{S}_{D}$, see (7.22), (7.24) (with $\tilde{\mathbf{r}}_{h}$ replaced by $\left.\mathbf{m}_{h}\right)$ and the facts that $\left.\left(\left(\nabla \mathbf{u}_{h}-\underline{\boldsymbol{\sigma}}_{h}\right) \mathbf{n}_{T}\right)\right|_{F}$ is in $\left[\mathbb{P}_{0}(F)\right]^{d}$ and that $\left.\llbracket \underline{\boldsymbol{\sigma}}_{h} \mathbf{n}_{F} \rrbracket\right|_{F}=0$ for all sides $F \in \partial \mathcal{S}_{D}^{\text {int }}$, we arrive at

$$
\begin{aligned}
& \left(\nabla \mathbf{u}_{h}-\underline{\boldsymbol{\sigma}}_{h}, \nabla \mathbf{m}_{h}\right)_{D} \\
& \quad=\sum_{T \in \mathcal{S}_{D}}\left\{-\left(\mathbf{m}_{h}, \nabla \cdot\left(\nabla \mathbf{u}_{h}-\underline{\boldsymbol{\sigma}}_{h}\right)\right)_{T}+\left\langle\left(\nabla \mathbf{u}_{h}-\underline{\boldsymbol{\sigma}}_{h}\right) \mathbf{n}_{T}, \mathbf{m}_{h}\right\rangle_{\partial T}\right\} \\
& =-\sum_{T \in \mathcal{S}_{D}}\left(\mathbf{m}_{h}, \mathbf{f}+\Delta \mathbf{u}_{h}-\nabla p_{h}\right)_{T}+\sum_{F \in \partial \mathcal{S}_{D}^{\text {int }}}\left\langle\llbracket \nabla \mathbf{u}_{h} \mathbf{n}_{F} \rrbracket, \mathbf{m}_{h}\right\rangle_{F} .
\end{aligned}
$$


We have also used that $\underline{\boldsymbol{\sigma}}_{h} \mathbf{n}_{F}=\nabla \mathbf{u}_{h} \mathbf{n}_{F}$ for all boundary sides $F$ of $\mathcal{S}_{D}$ not included in $\partial \Omega$ since $\underline{\boldsymbol{\sigma}}_{h} \in \underline{\boldsymbol{\Sigma}}_{\mathrm{N}}^{0}\left(\mathcal{S}_{D}\right)$, and (7.25) for all boundary sides $F$ of $\mathcal{S}_{D}$ included in $\partial \Omega$. By the Cauchy-Schwarz inequality and the inverse inequality $\left\|\mathbf{m}_{h}\right\|_{F} \lesssim h_{F}^{-\frac{1}{2}}\left\|\mathbf{m}_{h}\right\|_{T}$, we can further estimate

$$
\begin{aligned}
& \left(\nabla \mathbf{u}_{h}-\underline{\boldsymbol{\sigma}}_{h}, \nabla \mathbf{m}_{h}\right)_{D} \\
& \leq\left\{\sum_{T \in \mathcal{S}_{D}} h_{T}^{-2}\left\|\mathbf{m}_{h}\right\|_{T}^{2}\right\}^{1 / 2}\left\{\sum_{T \in \mathcal{S}_{D}} h_{T}^{2}\left\|\mathbf{f}+\Delta \mathbf{u}_{h}-\nabla p_{h}\right\|_{T}^{2}\right\}^{1 / 2} \\
& \quad+\left\{\sum_{F \in \partial \mathcal{S}_{D}^{\text {int }}} h_{F}^{-1}\left\|\mathbf{m}_{h}\right\|_{F}^{2}\right\}^{1 / 2}\left\{\sum_{F \in \partial \mathcal{S}_{D}^{\text {int }}} h_{F}\left\|\llbracket \nabla \mathbf{u}_{h} \mathbf{n}_{F} \rrbracket\right\|_{F}^{2}\right\}^{1 / 2} \\
& \lesssim h_{D}^{-1}\left\|\mathbf{m}_{h}\right\|_{D}\left\{\sum_{T \in \mathcal{S}_{D}} h_{T}^{2}\left\|\mathbf{f}+\Delta \mathbf{u}_{h}-\nabla p_{h}\right\|_{T}^{2}+\sum_{F \in \partial \mathcal{S}_{D}^{\text {int }}} h_{F}\left\|\llbracket \nabla \mathbf{u}_{h} \mathbf{n}_{F} \rrbracket\right\|_{F}^{2}\right\}^{1 / 2} .
\end{aligned}
$$

Recall that, as $\mathbf{m}_{h} \in \mathbf{M}\left(\mathcal{S}_{D}\right)$, we have (7.25) or (7.26) for $\mathbf{m}_{h}$. Thus, the discrete Poincaré/Friedrichs inequality

$$
\left\|\mathbf{m}_{h}\right\|_{D} \lesssim h_{D}\left\|\nabla \mathbf{m}_{h}\right\|_{D}
$$

can be easily proven along the lines of [58]. Consequently, (6.3) follows from the above estimates, the fact that $\llbracket p_{h} \underline{\boldsymbol{I}} \mathbf{n}_{F} \rrbracket=0$ for all $F \in \mathcal{S}_{D}^{\text {int }}$ since $p_{h} \in$ $C(\Omega)$, and (7.27).

\subsubsection{Higher-order continuous pressure elements}

The approach of the previous section does not generalize directly to higherorder conforming and conforming stabilized methods. When $\mathbf{V}_{h}$ contains piecewise polynomials of degree higher than 1 or $\mathbf{f}$ is not piecewise constant on $\mathcal{T}_{h}$, the local conservation property (7.15) does not hold. Here, we extend the approach of Section 7.2.1 to higher-order elements, namely the TaylorHood elements, the mini element, and higher-order stabilized methods.

Recall that each dual volume $D \in \mathcal{D}_{h}^{\text {int }}$ is associated with one vertex $V \in$ $\mathcal{V}_{h}^{\text {int }}$ and recall also the definition $\psi_{V, i}, i=1, \ldots, d$, of the vector Lagrange basis functions, see Section 7.2.1. For $V \in \mathcal{V}_{h}$, denote by $\partial \mathfrak{T}_{V}^{\text {int }}$ all the interior sides of the patch $\mathfrak{T}_{V}$. Then, choosing $\mathbf{v}_{h}=\boldsymbol{\psi}_{V, i}, i=1, \ldots, d$ as the test function in (7.11a) or (7.12a) (note that $\Delta \mathbf{v}_{h}$ becomes 0 in $(7.13 \mathrm{~b})$ ) and combining with the Green theorem, we obtain the following result:

Lemma 7.6 (Higher-order conforming methods on a dual mesh) Let $\left(\mathbf{u}_{h}, p_{h}\right) \in \mathbf{V}_{h} \times Q_{h}$ be given by (7.11a)-(7.11b) or by (7.12a)-(7.12b) with $\mathbf{V}_{h} \subset \mathbf{V}$ and $Q_{h} \subset H^{1}(\Omega)$. Let the normal flux functions $\boldsymbol{\Upsilon}_{F}\left(\mathbf{u}_{h}\right)$ be given by (7.14). Then

$$
\begin{aligned}
& \sum_{F \in \mathcal{F}_{D}}\left\langle\boldsymbol{\Upsilon}_{F}\left(\mathbf{u}_{h}\right) \mathbf{n}_{D} \cdot \mathbf{n}_{F}, \mathbf{e}_{i}\right\rangle_{F}-\left(\nabla p_{h}, \mathbf{e}_{i}\right)_{D}+\left(\mathbf{f}, \mathbf{e}_{i}\right)_{D} \\
& =\left(\mathbf{f}+\Delta \mathbf{u}_{h}-\nabla p_{h}, \mathbf{e}_{i}\right)_{D}-\left(\mathbf{f}+\Delta \mathbf{u}_{h}-\nabla p_{h}, \boldsymbol{\psi}_{V, i}\right) \mathfrak{T}_{V} \\
& \quad+\sum_{F \in \partial \mathfrak{T}_{V}^{\text {int }}}\left\langle\llbracket \nabla \mathbf{u}_{h} \mathbf{n}_{F} \rrbracket, \boldsymbol{\psi}_{V, i}\right\rangle_{F}-\sum_{F \in \partial \mathcal{S}_{D}^{\text {int }}}\left\langle\llbracket \nabla \mathbf{u}_{h} \mathbf{n}_{F} \rrbracket, \mathbf{e}_{i}\right\rangle_{F}, \\
& \quad i=1, \ldots, d, \quad \forall D \in \mathcal{D}_{h}^{\text {int }} .
\end{aligned}
$$


We know that lowest-order methods are locally conservative on the elements $D$ of $\mathcal{D}_{h}^{\text {int }}$, see (7.15). We can see from (7.29) that higher-order methods are not locally conservative because of the to additional terms featuring the element residuals $\mathbf{f}+\Delta \mathbf{u}_{h}-\nabla p_{h}$ and edge residuals $\llbracket \nabla \mathbf{u}_{h} \mathbf{n}_{F} \rrbracket$. We intend to redistribute these correction terms to the normal flux functions $\boldsymbol{\Upsilon}_{F}\left(\mathbf{u}_{h}\right)$ of (7.14) to obtain new normal flux functions $\boldsymbol{\Upsilon}_{F}\left(\mathbf{u}_{h}, p_{h}\right)$ satisfying

$$
\begin{array}{r}
\sum_{F \in \mathcal{F}_{D}}\left\langle\boldsymbol{\Upsilon}_{F}\left(\mathbf{u}_{h}, p_{h}\right) \mathbf{n}_{D} \cdot \mathbf{n}_{F}, \mathbf{e}_{i}\right\rangle_{F}-\left(\nabla p_{h}, \mathbf{e}_{i}\right)_{D}+\left(\mathbf{f}, \mathbf{e}_{i}\right)_{D}=0, \\
i=1, \ldots, d \quad \forall D \in \mathcal{D}_{h}^{\text {int }} .
\end{array}
$$

We achieve this in a spirit similar to the equilibration technique of [4]. As this represents a conceptually new technique for dual meshes $\mathcal{D}_{h}$-based a posteriori error estimates, which is of independent interest, we present it in Appendix B below.

Achieving, via Appendix B, (7.30), we can now proceed as in Section 7.2.1. Recall the definition of the space $\underline{\boldsymbol{\Sigma}}^{l}\left(\mathcal{S}_{D}\right)$ by $(7.5)$. For the new normal flux functions $\boldsymbol{\Upsilon}_{F}\left(\mathbf{u}_{h}, p_{h}\right)$ of (B6), we can define the space $\underline{\boldsymbol{\Sigma}}_{\mathrm{N}}^{l}\left(\mathcal{S}_{D}\right)$ by (7.19). We then set $\underline{\boldsymbol{\sigma}}_{h} \in \underline{\boldsymbol{\Sigma}}^{l}\left(\mathcal{S}_{h}\right)$ by (7.20) or, equivalently, by (7.21a)-(7.21b), where we put $l=k-1$. Note that the equation (7.30) holds for the new normal flux functions $\Upsilon_{F}\left(\mathbf{u}_{h}, p_{h}\right)$, whence the local Neumann problems are well-posed for $D \in \mathcal{D}_{h}^{\text {int }}$. We now have the following equivalent of Lemma 7.4:

Lemma 7.7 (Reconstructed flux in higher-order conforming methods) Let $\left(\mathbf{u}_{h}, p_{h}\right) \in \mathbf{V}_{h} \times Q_{h}$ be given by (7.11a)-(7.11b) or by (7.12a)(7.12b). Let $\boldsymbol{\Upsilon}_{F}\left(\mathbf{u}_{h}, p_{h}\right)$ be given by (B6) from Appendix B and prescribe $\underline{\boldsymbol{\sigma}}_{h}$ by (7.20). Then (5.9) holds. If $\mathbf{f}$ is piecewise polynomial of degree $l$ on $\overline{\mathcal{T}}_{h}$, then, more precisely,

$$
\left.\left(\nabla \cdot \underline{\boldsymbol{\sigma}}_{h}\right)\right|_{T}=\left.\left(\nabla p_{h}-\mathbf{f}\right)\right|_{T} \quad \forall T \in \mathcal{S}_{h} .
$$

Finally, we have:

Lemma 7.8 (Approximation property of the reconstructed flux in higher-order conforming methods) Let the assumptions of Lemma 7.7 be verified. Then the approximation property (6.3) holds.

Proof We proceed as in Lemma 7.5. Let $D \in \mathcal{D}_{h}$. Firstly, we need to replace the definition (7.23a)-(7.23b), valid in the lowest-order case, by defining $\tilde{\mathbf{r}}_{h} \in$ $\mathbf{M}\left(\mathcal{S}_{D}\right)$ by

$$
\begin{aligned}
\underline{\boldsymbol{\Pi}}_{\underline{\boldsymbol{\Sigma}}_{h}(T)}\left(\left.\nabla \tilde{\mathbf{r}}_{h}\right|_{T}\right) & =\left.\left(\underline{\boldsymbol{\sigma}}_{h}-\nabla \mathbf{u}_{h}\right)\right|_{T} & & \forall T \in \mathcal{S}_{D}, \\
\boldsymbol{\Pi}_{\mathbf{V}_{h}(T)}\left(\left.\tilde{\mathbf{r}}_{h}\right|_{T}\right) & =\left.\mathbf{r}_{h}\right|_{T} & & \forall T \in \mathcal{S}_{D} .
\end{aligned}
$$

Here $\underline{\boldsymbol{\Pi}}_{\underline{\boldsymbol{\Sigma}}_{h}(T)}$ is the $\underline{\boldsymbol{L}}^{2}$-orthogonal projection onto $\underline{\boldsymbol{\Sigma}}_{h}(T)$ and $\boldsymbol{\Pi}_{\mathbf{V}_{h}(T)}$ is the $\mathbf{L}^{2}$-orthogonal projection onto $\mathbf{V}_{h}(T)$. The space $\mathbf{M}\left(\mathcal{S}_{D}\right)$ is a vector variant of that of $[6,5]$. What is important in the present analysis is that by $(7.32 \mathrm{a})-$ (7.32b), the properties (7.24), (7.25), and (7.26) still hold. More precisely, the orthogonality in (7.24) and (7.25) holds up to polynomials of order $l$ in each component and not only for constants in each component. Similarly to $(7.27)$, we now have

$$
\left\|\nabla \mathbf{u}_{h}-\underline{\boldsymbol{\sigma}}_{h}\right\|_{D} \lesssim \sup _{\mathbf{m}_{h} \in \mathbf{M}\left(\mathcal{S}_{D}\right),\left\|\nabla \mathbf{m}_{h}\right\|_{D}=1}\left(\nabla \mathbf{u}_{h}-\underline{\boldsymbol{\sigma}}_{h}, \nabla \mathbf{m}_{h}\right)_{D}
$$


using $\left[62\right.$, Lemma 5.4]. Suppose now for simplicity that $\mathbf{f} \in\left[\mathbb{P}_{l}\left(\mathcal{S}_{h}\right)\right]^{d}$ instead of $\mathbf{f} \in\left[\mathbb{P}_{k}\left(\mathcal{S}_{h}\right)\right]^{d}$ required in Assumption 6.1. Then (7.28) still holds but with an additional factor

$$
-\sum_{F \in \partial \mathcal{S}_{D}^{\text {ext }}, F \not \subset \partial \Omega}\left\langle\boldsymbol{v}_{F}\left(\mathbf{u}_{h}, p_{h}\right) \mathbf{n}_{D} \cdot \mathbf{n}_{F}, \mathbf{m}_{h}\right\rangle_{F}
$$

in the last equality. We thus need to bound this factor, which we do by the Cauchy-Schwarz inequality and obtain

$$
\left\{\sum_{F \in \partial \mathcal{S}_{D}^{\text {ext }}, F \not \subset \partial \Omega} h_{F}^{-1}\left\|\mathbf{m}_{h}\right\|_{F}^{2}\right\}^{1 / 2}\left\{\sum_{F \in \partial \mathcal{S}_{D}^{\text {ext }}, F \not \subset \partial \Omega} h_{F}\left\|\boldsymbol{v}_{F}\left(\mathbf{u}_{h}, p_{h}\right)\right\|_{F}^{2}\right\}^{1 / 2} .
$$

The first term above can be treated as in Lemma 7.5 and we are left with bounding the second one.

Let $F \in \partial \mathcal{S}_{h}^{\text {int }}$, let $T \in \mathcal{T}_{h}$ be such that $F \subset T$, and let $V_{j}, D_{j}, j=$ $1, \ldots, d+1$, be the vertices of $T$ and the associated dual volumes. Using (B1), (B3), and (B4), we arrive at

$$
\begin{aligned}
& h_{F}^{1 / 2}\left\|\boldsymbol{v}_{F}\left(\mathbf{u}_{h}, p_{h}\right)\right\|_{F} \\
& \lesssim h_{F}^{1 / 2} \sum_{j=1}^{d+1} \sum_{i=1}^{d}\left\||F|^{-1}\left(\mathbf{f}+\Delta \mathbf{u}_{h}-\nabla p_{h},\left.\mathbf{e}_{i}\right|_{D_{j}}-\boldsymbol{\psi}_{V_{j}, i}\right)_{T}\right\|_{F} \\
& \quad+h_{F}^{1 / 2} \sum_{F^{\prime} \in \mathcal{F}_{T}^{\text {int }}} \sum_{j=1}^{d+1} \sum_{i=1}^{d}\left\||F|^{-1}\left\langle\llbracket \nabla \mathbf{u}_{h} \mathbf{n}_{F^{\prime}} \rrbracket,\left.\mathbf{e}_{i}\right|_{D_{j}}-\boldsymbol{\psi}_{V_{j}, i}\right\rangle_{F^{\prime}}\right\|_{F} .
\end{aligned}
$$

Let $i \in\{1, \ldots, d\}, j \in\{1, \ldots, d+1\}$, and $F^{\prime} \in \mathcal{F}_{T}^{\text {int }}$ be given. Then,

$$
\begin{aligned}
& h_{F}^{1 / 2}\left\||F|^{-1}\left(\mathbf{f}+\Delta \mathbf{u}_{h}-\nabla p_{h},\left.\mathbf{e}_{i}\right|_{D_{j}}-\boldsymbol{\psi}_{V_{j}, i}\right)_{T}\right\|_{F} \\
& \quad=h_{F}^{1 / 2}|F|^{-1 / 2}\left|\left(\mathbf{f}+\Delta \mathbf{u}_{h}-\nabla p_{h},\left.\mathbf{e}_{i}\right|_{D_{j}}-\boldsymbol{\psi}_{V_{j}, i}\right)_{T}\right| \\
& \quad \leq h_{F}^{1 / 2}|F|^{-1 / 2}\left\|\mathbf{f}+\Delta \mathbf{u}_{h}-\nabla p_{h}\right\|_{T}\left\|\left.\mathbf{e}_{i}\right|_{D_{j}}-\boldsymbol{\psi}_{V_{j}, i}\right\|_{T} \\
& \quad \lesssim h_{F}^{1 / 2}|F|^{-1 / 2}\left\|\mathbf{f}+\Delta \mathbf{u}_{h}-\nabla p_{h}\right\|_{T}|T|^{\frac{1}{2}} \\
& \quad \lesssim h_{T} \mid \mathbf{f}+\Delta \mathbf{u}_{h}-\nabla p_{h} \|_{T}
\end{aligned}
$$

by the Cauchy-Schwarz inequality and the facts that $\left\|\left.\mathbf{e}_{i}\right|_{D_{j}}-\boldsymbol{\psi}_{V_{j}, i}\right\|_{T} \lesssim|T|^{\frac{1}{2}}$, $|T|^{\frac{1}{2}} /|F|^{\frac{1}{2}} \lesssim h_{F}^{1 / 2}$, and $h_{F} \lesssim h_{T}$. Similarly,

$$
\begin{aligned}
& h_{F}^{1 / 2}\left\||F|^{-1}\left\langle\llbracket \nabla \mathbf{u}_{h} \mathbf{n}_{F^{\prime}} \rrbracket,\left.\mathbf{e}_{i}\right|_{D_{j}}-\boldsymbol{\psi}_{V_{j}, i}\right\rangle_{F^{\prime}}\right\|_{F} \\
& \quad=h_{F}^{1 / 2}|F|^{-1 / 2}\left|\left\langle\llbracket \nabla \mathbf{u}_{h} \mathbf{n}_{F^{\prime}} \rrbracket,\left.\mathbf{e}_{i}\right|_{D_{j}}-\boldsymbol{\psi}_{V_{j}, i}\right\rangle_{F^{\prime}}\right| \\
& \left.\quad \lesssim h_{F}^{1 / 2}|F|^{-1 / 2}\left\|\llbracket \nabla \nabla \mathbf{u}_{h} \mathbf{n}_{F^{\prime}} \rrbracket\right\|\left\|_{F^{\prime}}\right\| \mathbf{e}_{i}\right|_{D_{j}}-\boldsymbol{\psi}_{V_{j}, i} \|_{F^{\prime}} \\
& \quad \lesssim|F|^{-1 / 2}\left\|\llbracket \nabla \nabla \mathbf{u}_{h} \mathbf{n}_{F^{\prime}} \rrbracket\right\|_{F^{\prime}}\left\|\left.\mathbf{e}_{i}\right|_{D_{j}}-\boldsymbol{\psi}_{V_{j}, i}\right\|_{T} \\
& \quad \lesssim h_{F^{\prime}}^{1 / 2}\left\|\llbracket \nabla \nabla \mathbf{u}_{h} \mathbf{n}_{F^{\prime}} \rrbracket\right\|_{F^{\prime}},
\end{aligned}
$$

employing also the inverse inequality $\left\|\left.\mathbf{e}_{i}\right|_{D_{j}}-\psi_{V_{j}, i}\right\|_{F^{\prime}} \lesssim h_{F^{\prime}}^{-1 / 2} \|\left.\mathbf{e}_{i}\right|_{D_{j}}-$ $\boldsymbol{\psi}_{V_{j}, i} \|_{T}$. Combining all the above results, we arrive at the conclusion that the approximation property (6.3) holds in the higher-order case as well.

Remark 7.3 (Standard equilibration techniques) The equilibration techniques of $[4,27,14]$ can be used in order to produce $\underline{\boldsymbol{\sigma}}_{h} \in \underline{\boldsymbol{\Sigma}}^{l}\left(\mathcal{T}_{h}\right)$ satisfying $(5.9)$. Under the condition that (6.3) holds, they can likewise be used in the present framework. 


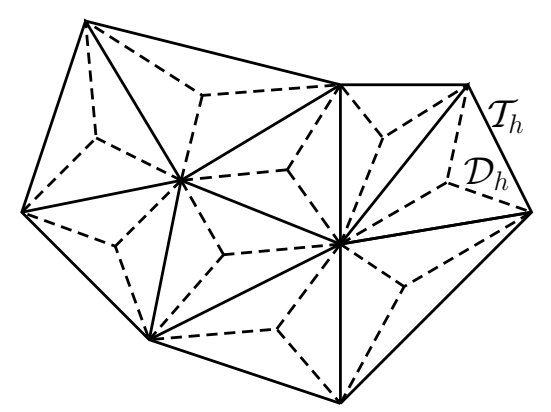

Fig. 7.2 Dual mesh $\mathcal{D}_{h}$ for the nonconforming Crouzeix-Raviart method in two space dimensions

\subsection{Nonconforming methods}

Here, we derive locally efficient a posteriori error estimates for the lowestorder nonconforming Crouzeix-Raviart method using Theorems 5.1 and 6.1. We follow the approach of Section 7.2.1. Extension to higher-order methods is possible along the lines of the approach of Section 7.2.2.

Let $\mathcal{T}_{h}$ be simplicial and matching and let

$$
\begin{aligned}
& \mathbf{V}_{h}:=\left\{\mathbf{v}_{h} \in\left[\mathbb{P}_{1}\left(\mathcal{T}_{h}\right)\right]^{d} ;\left\langle\llbracket \mathbf{v}_{h} \rrbracket, \mathbf{e}_{i}\right\rangle_{F}=0, \quad i=1, \ldots, d, \quad \forall F \in \partial \mathcal{T}_{h}\right\}, \\
& Q_{h}:=\mathbb{P}_{0}\left(\mathcal{T}_{h}\right) \cap Q .
\end{aligned}
$$

The lowest-order nonconforming Crouzeix-Raviart method for the problem (2.3a)-(2.3b) reads, see [25]: find $\left(\mathbf{u}_{h}, p_{h}\right) \in \mathbf{V}_{h} \times Q_{h}$ such that

$$
\begin{aligned}
a\left(\mathbf{u}_{h}, \mathbf{v}_{h}\right)+b\left(\mathbf{v}_{h}, p_{h}\right) & =\left(\mathbf{f}, \mathbf{v}_{h}\right) & & \forall \mathbf{v}_{h} \in \mathbf{V}_{h}, \\
b\left(\mathbf{u}_{h}, q_{h}\right) & =0 & & \forall q_{h} \in Q_{h} .
\end{aligned}
$$

Let the dual mesh $\mathcal{D}_{h}$ be formed around each side of $\mathcal{T}_{h}$ using the element barycenters as indicated in Figure $7.2 ; \mathcal{D}_{h}^{\text {int }}$ correspond to the interior sides and $\mathcal{D}_{h}^{\text {ext }}$ to the boundary ones. For a side $F \in \partial \mathcal{T}_{h}$, let $\psi_{F}$ be the $\mathbb{P}_{1}$ nonconforming finite element basis function. Let $\boldsymbol{\psi}_{F, i}, i=1, \ldots, d$, be its vector variants such that $\boldsymbol{\psi}_{F, i}^{i}=\psi_{F}, \boldsymbol{\psi}_{F, i}^{j}=0$ for $j=1, \ldots, d, j \neq i$. We will also need the fine simplicial mesh $\mathcal{S}_{h}$ formed by the $d+1$ subsimplices of each $T \in \mathcal{T}_{h}$, cf. once again Figure 7.2.

For a side $F \in \partial \mathcal{S}_{h}^{\text {int }}$ such that $F \subset \partial D$ for some $D \in \mathcal{D}_{h}$, define the normal flux functions

$$
\boldsymbol{\Upsilon}_{F}\left(\mathbf{u}_{h}, p_{h}\right):=\left(\nabla \mathbf{u}_{h}-p_{h} \underline{\boldsymbol{I}}\right) \mathbf{n}_{F} .
$$

Note that, as in the conforming setting of Section 7.2, all such sides lie inside some $T \in \mathcal{T}_{h}$, cf. Figure 7.2 , so that $\nabla \mathbf{u}_{h}$ and $p_{h}$ are indeed univalued thereon. holds.

As in Lemma 7.3 in the conforming case, the following important property

Lemma 7.9 (Local conservativity of the nonconforming CrouzeixRaviart method) Let $\mathbf{f}$ be piecewise constant on $\mathcal{T}_{h}$ and let $\left(\mathbf{u}_{h}, p_{h}\right) \in$ $\mathbf{V}_{h} \times Q_{h}$ be given by (7.33a)-(7.33b). Let $\boldsymbol{\Upsilon}_{F}\left(\mathbf{u}_{h}, p_{h}\right)$ be given by (7.34). Then

$$
\begin{array}{r}
\sum_{F \in \mathcal{F}_{D}}\left\langle\boldsymbol{\Upsilon}_{F}\left(\mathbf{u}_{h}, p_{h}\right) \mathbf{n}_{D} \cdot \mathbf{n}_{F}, \mathbf{e}_{i}\right\rangle_{F}+\left(\mathbf{f}, \mathbf{e}_{i}\right)_{D}=0, \\
i=1, \ldots, d, \quad \forall D \in \mathcal{D}_{h}^{\text {int }} .
\end{array}
$$


Proof For a given dual volume $D \in \mathcal{D}_{h}^{\text {int }}$ and associated side $F$, fix $i \in$ $\{1, \ldots, d\}$ and consider $\boldsymbol{\psi}_{F, i}$ as the test function $\mathbf{v}_{h}$ in $(7.33 \mathrm{a})$. Recall that the support of $\boldsymbol{\psi}_{F, i}$ is given by the two elements sharing $F$, denoted by $\mathcal{T}_{F}$. Then, under the assumption that $\mathbf{f}$ is piecewise constant on $\mathcal{T}_{h}$,

$$
\left(\mathbf{f}, \boldsymbol{\psi}_{F, i}\right)_{\mathcal{T}_{F}}=\left(\mathbf{f}, \mathbf{e}_{i}\right)_{D}
$$

easily follows as $|D \cap T|=|T| /(d+1)$ for all $T \in \mathcal{T}_{F}$. Next, consider $T \in \mathcal{T}_{F}$. One has

$$
\begin{aligned}
\left(\nabla \mathbf{u}_{h}, \nabla \boldsymbol{\psi}_{F, i}\right)_{T}= & \left(\nabla \mathbf{u}_{h}^{i}, \nabla \psi_{F}\right)_{T}=-\left(\Delta \mathbf{u}_{h}^{i}, \psi_{F}\right)_{T}+\left\langle\nabla \mathbf{u}_{h}^{i} \cdot \mathbf{n}_{T}, \psi_{F}\right\rangle_{\partial T} \\
= & \left\langle\nabla \mathbf{u}_{h}^{i} \cdot \mathbf{n}_{T}, \psi_{F}\right\rangle_{F}=\left\langle\nabla \mathbf{u}_{h}^{i} \cdot \mathbf{n}_{T}, 1\right\rangle_{F} \\
= & -\sum_{F^{\prime} \in \mathcal{F}_{D}, F^{\prime} \subset T}\left\langle\nabla \mathbf{u}_{h}^{i} \cdot \mathbf{n}_{D}, 1\right\rangle_{F^{\prime}} \\
=- & \sum_{F^{\prime} \in \mathcal{F}_{D}, F^{\prime} \subset T}\left\langle\nabla \mathbf{u}_{h} \mathbf{n}_{D}, \mathbf{e}_{i}\right\rangle_{F^{\prime}}
\end{aligned}
$$

using the fact that $\Delta \mathbf{u}_{h}^{i}=0$ as $\left.\mathbf{u}_{h}\right|_{T} \in\left[\mathbb{P}_{1}(T)\right]^{d}$, the facts that $\nabla \mathbf{u}_{h}^{i} \cdot \mathbf{n}_{T}$ is constant on all sides $F \in \mathcal{F}_{T}$, that $\left\langle 1, \psi_{F}\right\rangle_{F^{\prime}}=0$ for $F^{\prime} \in \mathcal{F}_{T}, F^{\prime} \neq F$, and that $\left\langle 1, \psi_{F}\right\rangle_{F}=\langle 1,1\rangle_{F}$, and finally once again the Green theorem and the fact that $\Delta \mathbf{u}_{h}^{i}=0$. Finally,

$$
\begin{aligned}
b\left(\boldsymbol{\psi}_{F, i}, p_{h}\right) & =-\left(\nabla \cdot \boldsymbol{\psi}_{F, i}, p_{h}\right)_{\mathcal{T}_{F}}=-\left(\nabla \boldsymbol{\psi}_{F, i}, p_{h} \underline{\boldsymbol{I}}\right)_{\mathcal{T}_{F}} \\
& =-\sum_{T \in \mathcal{T}_{F}}\left(\nabla \psi_{F}, \nabla\left(p_{h} \mathbf{x}^{i}\right)\right)_{T}=\sum_{F^{\prime} \in \mathcal{F}_{D}}\left\langle\nabla\left(p_{h} \mathbf{x}^{i}\right) \cdot \mathbf{n}_{D}, 1\right\rangle_{F^{\prime}} \\
& =\sum_{F^{\prime} \in \mathcal{F}_{D}}\left\langle p_{h} \mathbf{e}_{i} \cdot \mathbf{n}_{D}, 1\right\rangle_{F^{\prime}}=\sum_{F^{\prime} \in \mathcal{F}_{D}}\left\langle p_{h} \underline{\boldsymbol{I}} \mathbf{n}_{D}, \mathbf{e}_{i}\right\rangle_{F^{\prime}}
\end{aligned}
$$

by the same arguments as in (7.36) and using that $p_{h} \in \mathbb{P}_{0}\left(\mathcal{T}_{h}\right)$. Combining the above results, the assertion of the lemma follows.

We will now construct $\underline{\sigma}_{h}$ in the space $\underline{\Sigma}^{0}\left(\mathcal{S}_{h}\right)$, see $(7.5)$, on the fine simplicial mesh $\mathcal{S}_{h}$. For a given $D \in \mathcal{D}_{h}$ and $\boldsymbol{\Upsilon}_{F}\left(\mathbf{u}_{h}, p_{h}\right)$ given by (7.34), let

$$
\underline{\boldsymbol{\Sigma}}_{\mathrm{N}}^{0}\left(\mathcal{S}_{D}\right):=\left\{\underline{\boldsymbol{v}}_{h} \in \underline{\boldsymbol{\Sigma}}^{0}\left(\mathcal{S}_{D}\right) ; \underline{\boldsymbol{v}}_{h} \mathbf{n}_{F}=\boldsymbol{\Upsilon}_{F}\left(\mathbf{u}_{h}, p_{h}\right) \quad \forall F \in \partial \mathcal{S}_{h}^{\text {int }}, F \subset \partial D\right\} .
$$

We define $\underline{\boldsymbol{\sigma}}_{h} \in \underline{\boldsymbol{\Sigma}}^{0}\left(\mathcal{S}_{h}\right)$ by solving on each $D \in \mathcal{D}_{h}$ the following minimization problem:

$$
\left.\underline{\boldsymbol{\sigma}}_{h}\right|_{D}:=\arg \inf _{\underline{\boldsymbol{v}}_{h} \in \underline{\boldsymbol{\Sigma}}_{\mathrm{N}}^{0}\left(\mathcal{S}_{D}\right), \nabla \cdot \underline{\boldsymbol{v}}_{h}=-\boldsymbol{\Pi}_{0} \mathbf{f}}\left\|\nabla \mathbf{u}_{h}-p_{h} \underline{\boldsymbol{I}}-\underline{\boldsymbol{v}}_{h}\right\|_{D}
$$

Note that as we only have to set the normal fluxes over the side $F$ associated with the given $D \in \mathcal{D}_{h}$, the linear system (7.37), contrarily to (7.20), is trivial, with a diagonal $d \times d$ matrix; thus a direct flux formula follows from (7.37). We have the following result.

Lemma 7.10 (Reconstructed flux in the nonconforming CrouzeixRaviart method) Let $\mathbf{f}$ be piecewise constant on $\mathcal{T}_{h}$ and let $\left(\mathbf{u}_{h}, p_{h}\right) \in$ $\mathbf{V}_{h} \times Q_{h}$ be given by (7.33a)-(7.33b). Let $\underline{\boldsymbol{\sigma}}_{h}$ be defined by (7.37). Then (4.3) holds. More precisely,

$$
\left.\left(\nabla \cdot \underline{\boldsymbol{\sigma}}_{h}\right)\right|_{T}=-\left.\mathbf{f}\right|_{T} \quad \forall T \in \mathcal{S}_{h}
$$

Finally, the next result follows along the lines of the proof of Lemma 7.5.

Lemma 7.11 (Approximation property of the nonconforming Crouzeix-Raviart method) Let the assumptions of Lemma 7.10 be verified. Then the approximation property (6.2) holds. 
7.4 Finite volume and related locally conservative methods

This section is devoted to the application of the estimates of Theorems 5.1 and 6.1 to finite volume methods, cf., e.g., [30], and, in a larger sense, to general locally conservative methods such as the mimetic finite difference one, cf., e.g., [11].

A general locally conservative method for the problem (2.3a)-(2.3b) enables to find the side normal fluxes $\boldsymbol{\Upsilon}_{F}$, constant $d$-dimensional vectors for each side $F \in \partial \mathcal{T}_{h}$, such that

$$
\sum_{F \in \mathcal{F}_{T}} \boldsymbol{\Upsilon}_{F}^{i}\left(\mathbf{n}_{T} \cdot \mathbf{n}_{F}\right)+\left(\mathbf{f}, \mathbf{e}_{i}\right)_{T}=0, \quad i=1, \ldots, d, \quad \forall T \in \mathcal{T}_{h}
$$

Usually, velocities $\mathbf{u}_{h} \in\left[\mathbb{P}_{0}\left(\mathcal{T}_{h}\right)\right]^{d}$ and pressures $p_{h} \in \mathbb{P}_{0}\left(\mathcal{T}_{h}\right)$ are also obtained from the given numerical scheme.

Suppose first that $\mathcal{T}_{h}$ is simplicial and matching. In order to obtain a posteriori error estimates in this case, we define a flux (stress) $\underline{\boldsymbol{\sigma}}_{h}$ in the Raviart-Thomas-Nédélec space $\underline{\boldsymbol{\Sigma}}^{0}\left(\mathcal{T}_{h}\right)$, see (7.5), prescribing its degrees of freedom by

$$
\left.\underline{\boldsymbol{\sigma}}_{h} \mathbf{n}_{F}\right|_{F}=\frac{\boldsymbol{\Upsilon}_{F}}{|F|} \quad \forall F \in \mathcal{F}_{T}, \quad \forall T \in \mathcal{T}_{h}
$$

Then (4.3) immediately follows from (7.39), (7.40), and the Green theorem. Consequently, Theorem 5.1 could directly be applied to obtain an a posteriori error estimate for $\left\|\left(\mathbf{u}-\mathbf{u}_{h}, p-p_{h}\right)\right\| \|$. As, however, $\mathbf{u}_{h} \in\left[\mathbb{P}_{0}\left(\mathcal{T}_{h}\right)\right]^{d}, \nabla \mathbf{u}_{h}$ is a zero tensor and such an estimate would be of very little practical value. We thus, following $[59,61]$, introduce a postprocessed velocity $\tilde{\mathbf{u}}_{h} \in\left[\mathbb{P}_{2}\left(\mathcal{T}_{h}\right)\right]^{d}$ satisfying

$$
\begin{aligned}
\left.\nabla \tilde{\mathbf{u}}_{h}\right|_{T}-\left.p_{h} \underline{\boldsymbol{I}}\right|_{T} & =\left.\underline{\boldsymbol{\sigma}}_{h}\right|_{T} \quad \forall T \in \mathcal{T}_{h}, \\
\frac{\left(\tilde{\mathbf{u}}_{h}, \mathbf{e}_{i}\right)_{T}}{|T|} & =\left.\mathbf{u}_{h}^{i}\right|_{T}, \quad i=1, \ldots, d, \quad \forall T \in \mathcal{T}_{h} .
\end{aligned}
$$

Note that such a postprocessing is local on each mesh element $T$ and is cheap, as we merely prescribe the degrees of freedom of $\tilde{\mathbf{u}}_{h}$. The advantage of this postprocessing is twofold: firstly, $\nabla \tilde{\mathbf{u}}_{h}$ is no more a zero tensor and it gives a good sense to estimate $\left\|\left(\mathbf{u}-\tilde{\mathbf{u}}_{h}, p-p_{h}\right)\right\|$; secondly, by (7.41a), $\left\|\nabla \tilde{\mathbf{u}}_{h}-p_{h} \underline{\boldsymbol{I}}-\underline{\boldsymbol{\sigma}}_{h}\right\|_{T}=0$. Thus, (6.2) (with $\mathbf{u}_{h}$ replaced by $\tilde{\mathbf{u}}_{h}$ ) is trivially satisfied. This is perfectly in agreement with the "flux-conforming" nature of locally conservative methods.

Meshes consisting of general polygons (polyhedrons), possibly nonconvex and not star-shaped, and nonmatching meshes can be taken into account following [61, Section 5]: one introduces a simplicial submesh $\mathcal{S}_{T}$ of each $T \in$ $\mathcal{T}_{h}$ such that $\mathcal{S}_{T}$ form a conforming simplicial mesh $\mathcal{S}_{h}$ of $\Omega$. One then uses the validity of the balance equation (7.39) on each $T \in \mathcal{T}_{h}$ in order to solve on the mesh $\mathcal{S}_{T}$ of each $T \in \mathcal{T}_{h}$ a local Stokes problem, yielding a side normal flux $\boldsymbol{\Upsilon}_{F}$ for each side $F \in \partial \mathcal{S}_{h}$ and $\mathbf{u}_{h} \in\left[\mathbb{P}_{0}\left(\mathcal{S}_{h}\right)\right]^{d}$ and $p_{h} \in \mathbb{P}_{0}\left(\mathcal{S}_{h}\right)$. Then the approach of the previous paragraph can be applied in a straightforward way on the mesh $\mathcal{S}_{h}$.

Remark 7.4 (Estimates for the fluxes $\underline{\boldsymbol{\sigma}}_{h}$ ) Estimates on the error directly in the fluxes $\underline{\boldsymbol{\sigma}}_{h}$ can be established along the lines of the analysis in [62]. 
7.5 Mixed finite element methods

Here, we derive locally efficient a posteriori error estimates for mixed finite element methods using Corollary 5.1 and Theorem 6.1. We suppose that $\mathcal{T}_{h}$ is simplicial and matching.

The mixed finite element method for problem (2.3a)-(2.3b) reads: find $\left(\underline{\boldsymbol{\sigma}}_{h}, \mathbf{u}_{h}, p_{h}\right) \in \underline{\boldsymbol{\Sigma}}_{h} \times \mathbf{V}_{h} \times Q_{h}$, the approximation to the stress tensor $\underline{\boldsymbol{\sigma}}$, the velocity $\mathbf{u}$, and the pressure $p$, respectively, such that

$$
\begin{aligned}
\left(\underline{\boldsymbol{\sigma}}_{h}, \underline{\boldsymbol{\tau}}_{h}\right)+\left(\mathbf{u}_{h}, \nabla \cdot \boldsymbol{\tau}_{h}\right) & =0 & & \forall \underline{\boldsymbol{\tau}}_{h} \in \underline{\boldsymbol{\Sigma}}_{h}, \\
-\left(\nabla \cdot \underline{\boldsymbol{\sigma}}_{h}, \mathbf{v}_{h}\right)+\left(\nabla p_{h}, \mathbf{v}_{h}\right) & =\left(\mathbf{f}, \mathbf{v}_{h}\right) & & \forall \mathbf{v}_{h} \in \mathbf{V}_{h}, \\
\left(\mathbf{u}_{h}, \nabla q_{h}\right) & =0 & & \forall q_{h} \in Q_{h} .
\end{aligned}
$$

We consider the Raviart-Thomas-Nédélec spaces $\underline{\Sigma}_{h}:=\underline{\Sigma}^{k}\left(\mathcal{T}_{h}\right)$ (see (7.5)), $\mathbf{V}_{h}:=\left[\mathbb{P}_{k}\left(\mathcal{T}_{h}\right)\right]^{d}$, and $Q_{h}:=\mathbb{P}_{k+1}\left(\mathcal{T}_{h}\right) \cap C(\Omega) \cap \bar{Q}, k \geq 0$. Brezzi-Douglas Marini/Brezzi-Douglas-Durán-Fortin finite element spaces can also be considered, as in [53].

In order to obtain an upper bound on the error $\left\|\left(\mathbf{u}-\mathbf{u}_{h}, p-p_{h}\right)\right\| \mid$, we could now directly apply Corollary 5.1. Indeed, $\underline{\boldsymbol{\sigma}}_{h} \in \underline{\boldsymbol{\Sigma}}_{h}$, so that $\underline{\boldsymbol{\sigma}}_{h}$ belongs to $\underline{\boldsymbol{H}}(\operatorname{div}, \Omega)$ by definition, and (5.9) follows from (7.42b). As, however, explained in Section 7.4, such a direct application is not too wise. Thus, following $[6,5,59,62]$, we once again introduce a cheap elementwise postprocessing of the velocity $\mathbf{u}_{h}$. Let $T \in \mathcal{T}_{h}$ and let $\underline{\boldsymbol{\Sigma}}_{h}(T)$ denote the restriction of $\underline{\boldsymbol{\Sigma}}_{h}$ onto $T$ and similarly for $\mathbf{V}_{h}(T)$. We look for $\left.\tilde{\mathbf{u}}_{h}\right|_{T} \in \mathbf{M}_{h}(T)$ such that

$$
\begin{array}{rlrl}
\left(\nabla \tilde{\mathbf{u}}_{h}-\underline{\boldsymbol{\sigma}}_{h}, \underline{\boldsymbol{\tau}}_{h}\right)_{T} & =0 & \forall \underline{\boldsymbol{\tau}}_{h} \in \underline{\boldsymbol{\Sigma}}_{h}(T), \\
\left(\tilde{\mathbf{u}}_{h}-\mathbf{u}_{h}, \mathbf{v}_{h}\right)=0 & \forall \mathbf{v}_{h} \in \mathbf{V}_{h}(T) .
\end{array}
$$

Equivalently, the above definition can be expressed as

$$
\begin{aligned}
\underline{\boldsymbol{\Pi}}_{\underline{\boldsymbol{\Sigma}}_{h}(T)}\left(\left.\nabla \tilde{\mathbf{u}}_{h}\right|_{T}\right) & =\left.\underline{\boldsymbol{\sigma}}_{h}\right|_{T}, \\
\boldsymbol{\Pi}_{\mathbf{V}_{h}(T)}\left(\left.\tilde{\mathbf{u}}_{h}\right|_{T}\right) & =\left.\mathbf{u}_{h}\right|_{T},
\end{aligned}
$$

where $\underline{\boldsymbol{\Pi}}_{\underline{\boldsymbol{\Sigma}}_{h}(T)}$ is the $\underline{\boldsymbol{L}}^{2}$-orthogonal projection onto $\underline{\boldsymbol{\Sigma}}_{h}(T)$ and $\boldsymbol{\Pi}_{\mathbf{V}_{h}(T)}$ is the $\mathbf{L}^{2}$-orthogonal projection onto $\mathbf{V}_{h}(T)$. The spaces $\mathbf{M}_{h}(T)$ are vector variants of those of $[6,5]$. They are typically $\left[\mathbb{P}_{k+1}\left(\mathcal{T}_{h}\right)\right]^{d}$ spaces enriched by bubbles. Moreover, in the lowest-order case $(k=0)$, as in (7.41a)-(7.41b) (cf. also (7.23a)-(7.23b)), following [59], one can easily build $\tilde{\mathbf{u}}_{h}$ such that

$$
\begin{array}{rlrl}
\left.\nabla \tilde{\mathbf{u}}_{h}\right|_{T} & =\left.\underline{\boldsymbol{\sigma}}_{h}\right|_{T} & & \forall T \in \mathcal{T}_{h}, \\
\frac{\left(\tilde{\mathbf{u}}_{h}, \mathbf{e}_{i}\right)_{T}}{|T|} & =\left.\mathbf{u}_{h}^{i}\right|_{T}, \quad & i=1, \ldots, d, \quad \forall T \in \mathcal{T}_{h} .
\end{array}
$$

We then apply Corollary 5.1 in order to estimate $\left\|\left(\mathbf{u}-\tilde{\mathbf{u}}_{h}, p-p_{h}\right)\right\| \mid$. In the lowest-order case $(k=0)$ and constructing $\tilde{\mathbf{u}}_{h}$ by $(7.45 \mathrm{a})-(7.45 \mathrm{~b}), \| \nabla \tilde{\mathbf{u}}_{h}-$ $\underline{\boldsymbol{\sigma}}_{h} \|_{T}=0$. Hence in this case, (6.3) is trivially satisfied, once again in agreement with the flux-conforming nature of mixed finite elements. For $k \geq 1$, this property does not hold exactly anymore. By (7.44a), however, $\left\|\nabla \tilde{\mathbf{u}}_{h}-\underline{\boldsymbol{\sigma}}_{h}\right\|_{T}$ is expected to be small and act as a numerical quadrature. Finally, we note that Remark 7.4 applies here as well. Proceeding as in [62], rigorous bothsided estimates, also including the estimates on the error directly in the fluxes $\underline{\sigma}_{h}$, can be obtained. 


\section{Numerical experiments}

In this section, we illustrate the theory on numerical experiments using discontinuous, conforming, and nonconforming methods. As a discontinuous method, we consider first- and second-order symmetric discontinuous Galerkin (DG) method of Section 7.1, i.e., (7.2) with $\theta=1$ and $k=1,2$. The conforming example will be computed using the $\mathbb{P}_{1}$ iso $\mathbb{P}_{2}-\mathbb{P}_{1}$ method of Section 7.2 and the nonconforming example using the Crouzeix-Raviart method of Section 7.3.

The a posteriori error estimates for these methods are obtained by recovering the equilibrated flux $\underline{\boldsymbol{\sigma}}_{h}$ and applying Theorem 5.1 or Corollary 5.1, depending on the method. For the discontinuous Galerkin method of order $k$, we recover the flux from the space $\underline{\Sigma}^{k}\left(\mathcal{T}_{h}\right)$. The error estimator is obtained by applying Theorem 5.1. For such flux and sufficiently regular f, Lemma 7.1 guarantees superconvergence for the residual error estimators $\eta_{\mathrm{R}, T}$ of (5.3) by two orders of magnitude. As we will see, this is not true if $\mathbf{f}$ is not sufficiently regular. For the $\mathbb{P}_{1}$ iso $\mathbb{P}_{2}-\mathbb{P}_{1}$ and Crouzeix-Raviart methods, the flux is recovered by solving local minimization problems (7.20) and (7.37), respectively. In order for Lemmas 7.4 and 7.10 hold, we, as usual, implement these methods with $\mathbf{f}$ replaced by $\boldsymbol{\Pi}_{0} \mathbf{f}$, where $\boldsymbol{\Pi}_{0}$ is the $\mathbf{L}^{2}$-orthogonal projection onto $\left[\mathbb{P}_{0}\left(\mathcal{T}_{h}\right)\right]^{d}$. We then include the data oscillation in the residual error estimators $\eta_{\mathrm{R}, T}(5.7)$ and (5.3) as $C_{\mathrm{P}, T} h_{T}\left\|\mathbf{f}-\boldsymbol{\Pi}_{0} \mathbf{f}\right\|_{T}$, which are also superconvergent (by one order of magnitude) for smooth $\mathbf{f}$. The error estimate for the $\mathbb{P}_{1}$ iso $\mathbb{P}_{2}-\mathbb{P}_{1}$ method is obtained by applying Corollary 5.1 and for the Crouzeix-Raviart method by applying Theorem 5.1.

Throughout this section, we will consider domain $\Omega=(0,1)^{2}$. To evaluate the energy (semi-)norm (3.5) and the divergence error estimator $\eta_{\mathrm{D}, T}$ of (5.2) the inf-sup constant $\beta$ has to be estimated. Although the inf-sup constant can be estimated analytically for rectangular domains, we have computed $\beta$ with the procedure from [28]. Based on these computations, the value $\beta=0.44$ is used.

The load function $\mathbf{f}$ is chosen to correspond to the solution

$$
\mathbf{u}=\nabla \times(x-1)^{2} x^{1+\alpha}(y-1)^{2} y^{2} \mathbf{e}_{3}, \quad p=x+y-1 .
$$

For $\alpha>0$, the velocity field $\mathbf{u}$ has a zero divergence, $\nabla \cdot \mathbf{u}=0$, and satisfies the zero Dirichlet boundary condition, $\mathbf{u}=0$ on $\partial \Omega$. The regularity of $\mathbf{u}$ is $\left[H^{\frac{1}{2}+\alpha}(\Omega)\right]^{d}$ for $\alpha \notin \mathbb{N}$ and $\left[C^{\infty}(\Omega)\right]^{d}$ for $\alpha \in \mathbb{N}$.

For all methods, we will first consider problem with a smooth solution. For this purpose, the parameter $\alpha$ is chosen as $\alpha=1$. To compare the error estimator with the exact error in uniform refinement, we have solved the problem at hand with each of the mentioned methods on a set of uniformly refined meshes. The error and estimates behavior for the different schemes are visualized in Figure 8.1. The corresponding effectivity indices, given as the ratios of the estimate over the error, are presented in Figure 8.2. The different estimators, namely

$$
\left\{\sum_{T \in \mathcal{T}_{h}} \eta_{T}^{2}\right\}^{1 / 2}
$$

with $\eta_{T}=\eta_{\mathrm{NC}, T}, \eta_{\mathrm{DF}, T} / C_{\mathrm{S}}, \eta_{\mathrm{R}, T} / C_{\mathrm{S}}$, and $\eta_{\mathrm{D}, T} / C_{\mathrm{S}}$, are plotted in Figure 8.3. For each method, the predicted superconvergence for the $\eta_{\mathrm{R}, T}$ part is observed. 

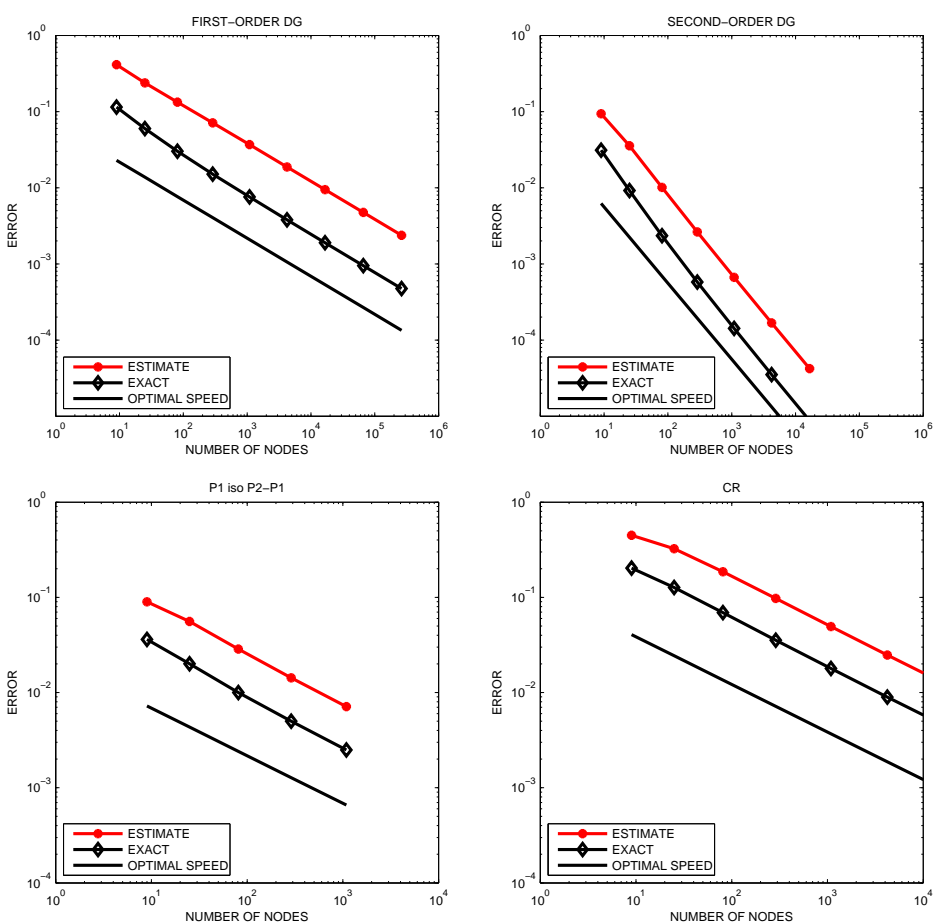

Fig. 8.1 Estimated and exact errors for the first-order DG, second-order DG, $\mathbb{P}_{1}$ iso $\mathbb{P}_{2}-\mathbb{P}_{1}$, and Crouzeix-Raviart methods for the smooth test case

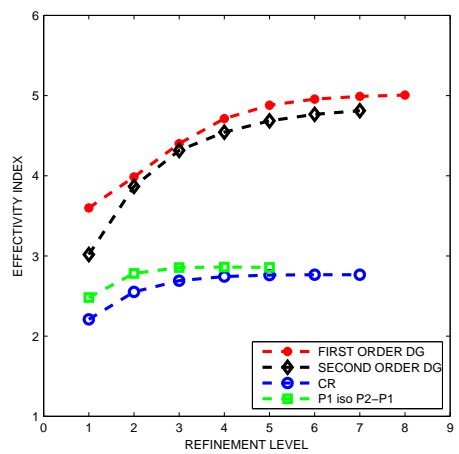

Fig. 8.2 Effectivity indices for the first-order DG, second-order $D G, \mathbb{P}_{1}$ iso $\mathbb{P}_{2}-\mathbb{P}_{1}$, and Crouzeix-Raviart methods for the smooth test case

The error distributions from refinement step 5 for discontinuous Galerkin methods, the $\mathbb{P}_{1}$ iso $\mathbb{P}_{2}-\mathbb{P}_{1}$ method, and the Crouzeix-Raviart method are given respectively in Figures 8.4, 8.5, 8.6, and 8.7. As the error bounds given in Theorem 5.1 or Corollary 5.1 are not in an elementwise form, we have estimated the upper bound as

$$
\left\|\left(\mathbf{u}-\mathbf{u}_{h}, p-p_{h}\right)\right\|^{2} \leq 2 \sum_{T \in \mathcal{T}_{h}}\left\{\eta_{\mathrm{NC}, T}^{2}+\frac{1}{C_{\mathrm{S}}^{2}}\left(\eta_{\mathrm{R}, T}+\eta_{\mathrm{DF}, T}\right)^{2}+\eta_{\mathrm{D}, T}^{2}\right\} .
$$

We have used the term $\eta_{T}:=\left\{2\left(\eta_{\mathrm{NC}, T}^{2}+C_{\mathrm{S}}^{-2}\left(\eta_{\mathrm{R}, T}+\eta_{\mathrm{DF}, T}\right)^{2}+\eta_{\mathrm{D}, T}^{2}\right)\right\}^{1 / 2}$ as elementwise error estimator. For comparison of the exact and estimated error 

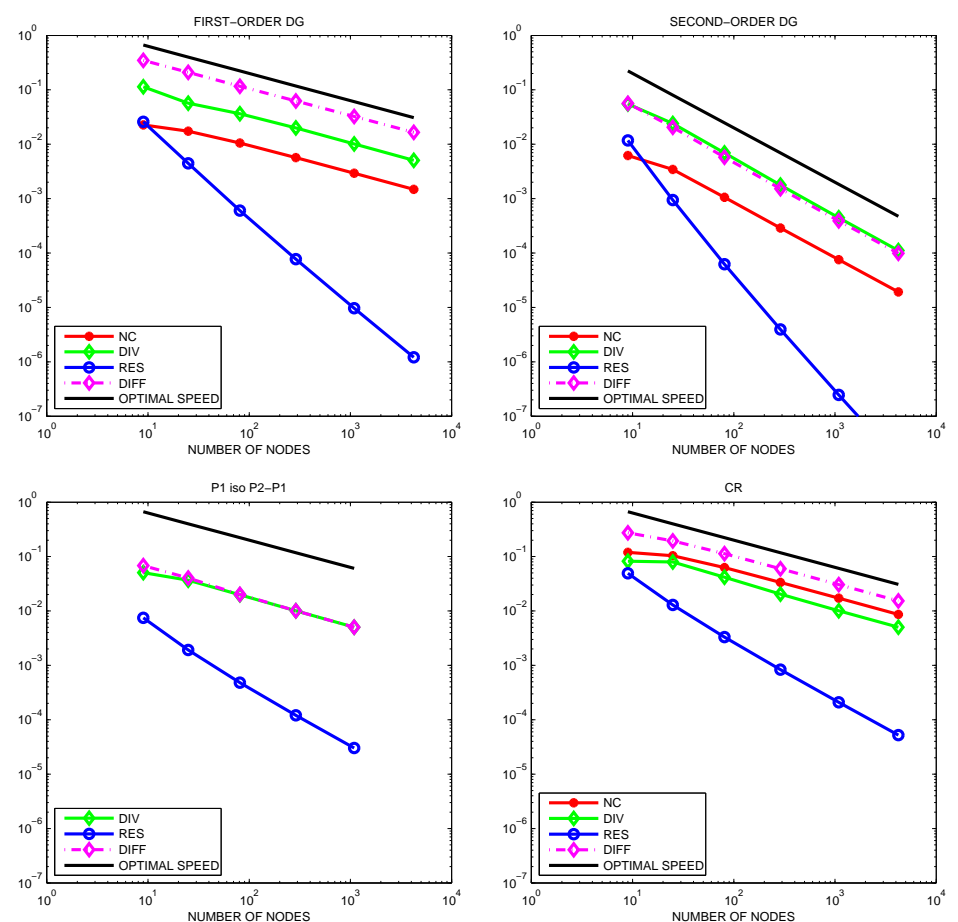

Fig. 8.3 Different estimators for the first-order DG, second-order DG, $\mathbb{P}_{1}$ iso $\mathbb{P}_{2}-$ $\mathbb{P}_{1}$, and Crouzeix-Raviart methods for the smooth test case
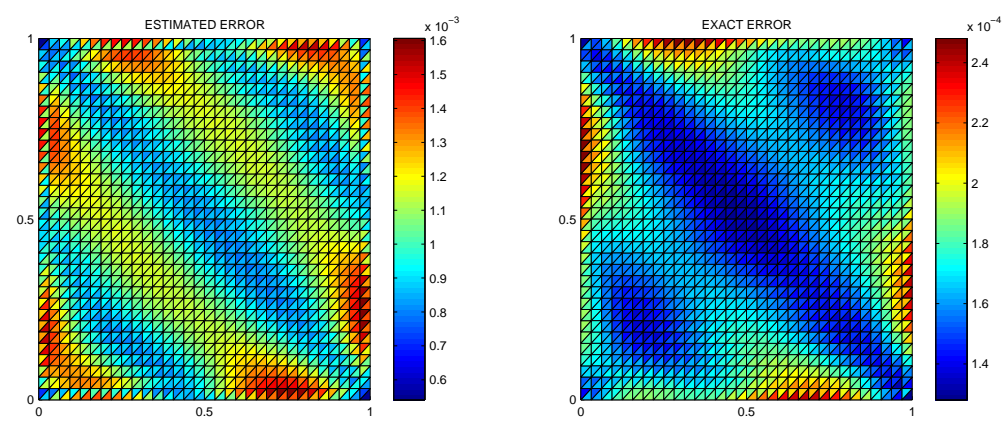

Fig. 8.4 Estimated (left) and exact (right) error distributions for the first-order DG method in the smooth test case

distributions, we have also included local the effectivity indices in Figure 8.8. Based on our results, we can conclude that the predicted and exact error distributions match quite well.

In the second example, we set $\alpha=0.75$, so that the velocity $\mathbf{u}$ is in $\left[H^{1.25}(\Omega)\right]^{d}$ and there is a boundary singularity on the edge $x=0$. In this example, all computations were performed using the first-order DG method. The problem was solved either on uniformly refined meshes or using a simple adaptive procedure. In the adaptive routine, we refine ten percents of elements in each step using the Matlab PDE toolbox refinemesh algorithm. The elements are chosen such that they have the largest element estimators $\eta_{T}$. 

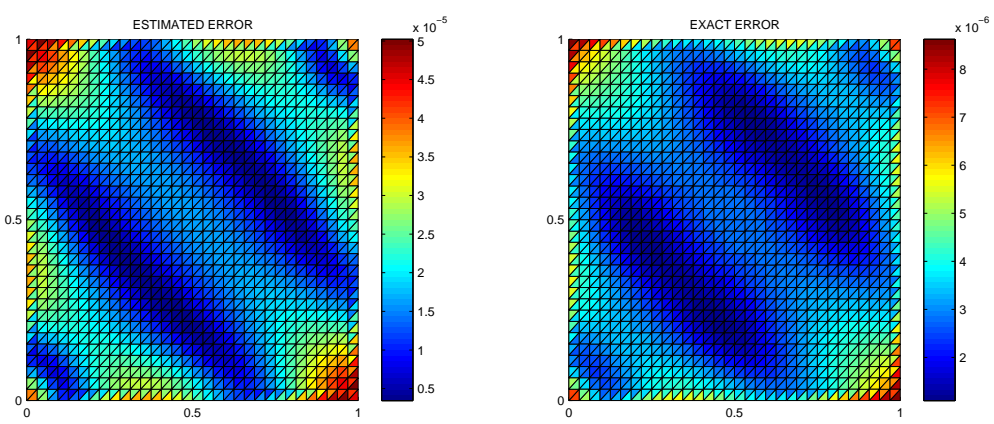

Fig. 8.5 Estimated (left) and exact (right) error distributions for the second-order DG method in the smooth test case
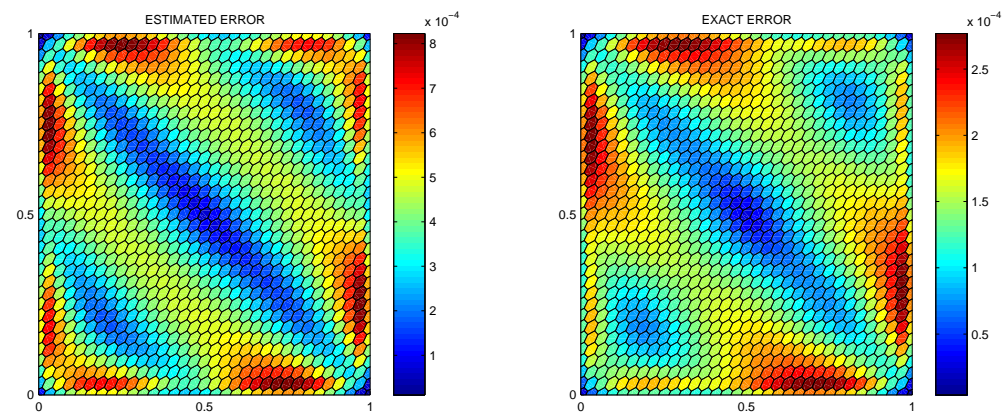

Fig. 8.6 Estimated (left) and exact (right) error distributions for the $\mathbb{P}_{1}$ iso $\mathbb{P}_{2}-\mathbb{P}_{1}$ method in the smooth test case
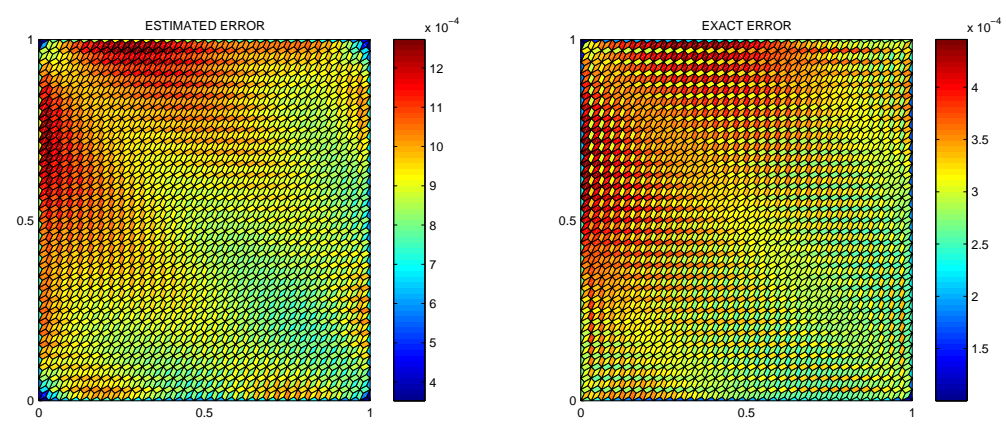

Fig. 8.7 Estimated (left) and exact (right) error distributions for the nonconforming Crouzeix-Raviart method in the smooth test case

The error behavior in the adaptive and uniform refinement procedures is visualized in Figure 8.10 and the corresponding effectivity index in the adaptive case in Figure 8.10, left. The uniform mesh refinement displays the convergence rate $\mathcal{O}\left(h^{0.25}\right)$, which is in agreement with the $\left[H^{1.25}(\Omega)\right]^{d}$ regularity of the weak solution. The adaptive refinement procedure has considerably faster convergence rate. However, the optimal convergence rate is not achieved even with the adaptive solution strategy. This is probably due to 

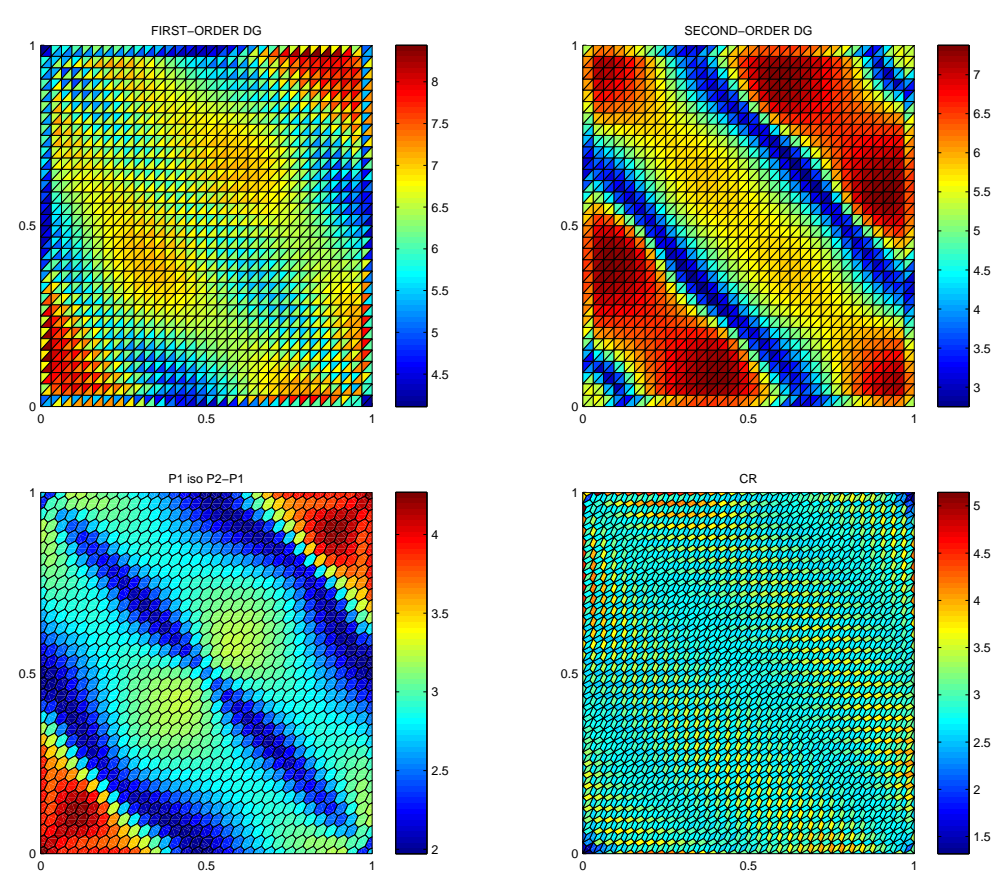

Fig. 8.8 Local effectivity indices for the error distributions
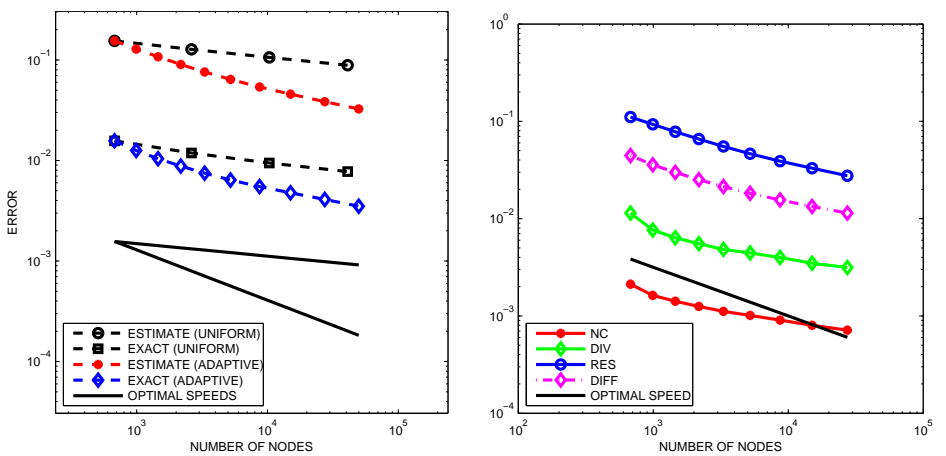

Fig. 8.9 Estimated and exact errors in uniform/adaptive refinement (left) and components of the error estimator in adaptive refinement (right) for the first-order DG method in the singular test case

the difficult nature of the problem. In order to exclude that this phenomenon is caused by our error estimator, we include Figure 8.10, right. Here we give a comparison of the adaptive refinement process when driven by our a posteriori error estimate and when done following the exact distribution of the error (known herein). Clearly, the exact error behavior is not dependent on the applied error distribution used to drive the adaptivity. As in the smooth test case, the error estimate overestimates the error, but decreases with the same speed as the exact error.

Figure 8.11 shows the estimated and exact error distributions in the singular test case. They once again match quite well; in particular the boundary singularity is well detected. The superconvergence of $\eta_{\mathrm{R}, T}$ does not appear 

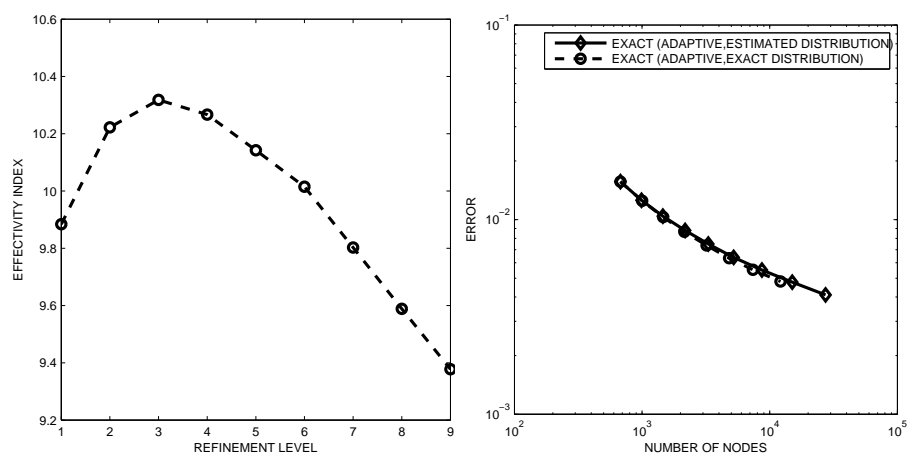

Fig. 8.10 Effectivity indices for the first-order DG method in the singular test case (left) and exact error in the adaptive process using estimated and exact error distributions (right)
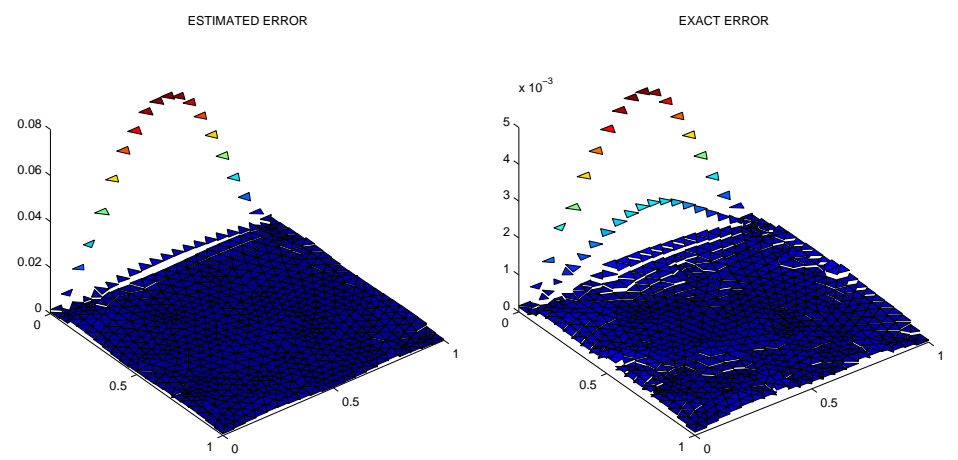

Fig. 8.11 Estimated (left) and exact (right) error distributions for the first-order DG method in the singular test case

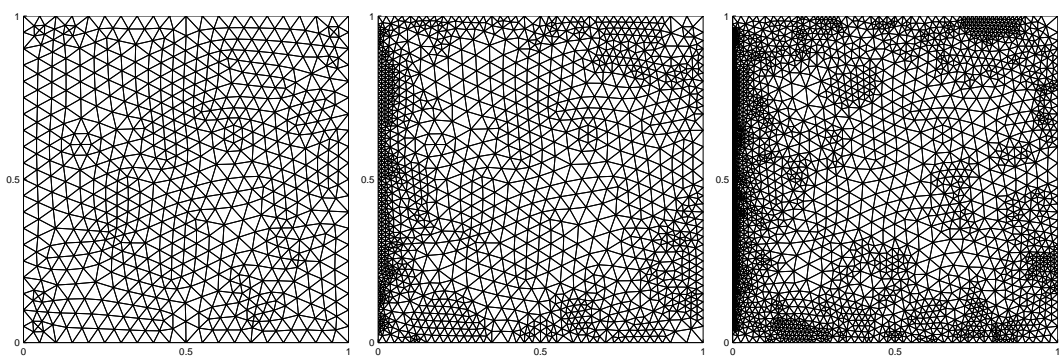

Fig. 8.12 Initial (left), third (middle), and fifth (right) mesh for the adaptive refinement for the first-order DG method in the singular test case

anymore as $\mathbf{f}$ is not sufficiently regular. For the sake of completeness, initial, third, and fifth adaptive mesh are visualized in Figure 8.12. One can observe the expected refinement towards the boundary singularity. 


\section{Appendix}

\section{A Characterization of the inf-sup constants}

In this section we will show a proof of Lemma 3.1, following the ideas of [46]. We start by the following well-known result $[38,55]$ :

Lemma A.1 (Characterization of the inf-sup constant) The inf-sup constant $\beta$ of (2.4) is the square root of the smallest eigenvalue to the following generalized eigenvalue problem

$$
\begin{aligned}
a(\mathbf{u}, \mathbf{v})+b(\mathbf{v}, p) & =0 & & \forall \mathbf{v} \in \mathbf{V}, \\
b(\mathbf{u}, q) & =-\lambda(p, q) & & \forall q \in Q .
\end{aligned}
$$

Proof Define the following operators

$$
\begin{aligned}
& A:=-\Delta: \mathbf{V} \rightarrow \mathbf{V}^{*}, \\
& B:=\nabla: Q \rightarrow \mathbf{V}^{*},
\end{aligned}
$$

so that it holds

$$
a(\mathbf{v}, \mathbf{v})=\left(A^{1 / 2} \mathbf{v}, A^{1 / 2} \mathbf{v}\right) \quad \text { and } \quad b(\mathbf{v}, q)=\left(B^{*} \mathbf{v}, q\right)
$$

for all $\mathbf{v} \in \mathbf{V}$ and all $q \in Q$. With this notation, we have

$$
\frac{b(\mathbf{v}, q)}{\|\nabla \mathbf{v}\|\|q\|}=\frac{\left(B^{*} \mathbf{v}, q\right)}{\left\|A^{1 / 2} \mathbf{v}\right\|\|q\|}
$$

Substituting $\mathbf{z}:=A^{1 / 2} \mathbf{v}$ gives

$$
\frac{b(\mathbf{v}, q)}{\|\nabla \mathbf{v}\|\|q\|}=\frac{\left(B^{*} A^{-1 / 2} \mathbf{z}, q\right)}{\|\mathbf{z}\|\|q\|}=\frac{\left(\mathbf{z}, A^{-1 / 2} B q\right)}{\|\mathbf{z}\|\|q\|}
$$

and hence the supremum is obtained by choosing

$$
\mathbf{z}=\frac{A^{-1 / 2} B q}{\left\|A^{-1 / 2} B q\right\|}
$$

and we come to

$$
\beta=\inf _{q \in Q} \frac{\left\|A^{-1 / 2} B q\right\|}{\|q\|}
$$

Squaring gives

$$
\beta^{2}=\inf _{q \in Q} \frac{\left\|A^{-1 / 2} B q\right\|^{2}}{\|q\|^{2}} .
$$

This is the Rayleigh quotient for the eigenvalue problem

$$
B^{*} A^{-1} B p=\lambda p .
$$

Denoting $\mathbf{u}=-A^{-1} B p$ this is written as

$$
\begin{gathered}
A \mathbf{u}+B p=0, \\
B^{*} \mathbf{u}=-\lambda p,
\end{gathered}
$$

i.e., the operator form of (A.1).

We are now ready to prove Lemma 3.1.

Proof (Proof of Lemma 3.1) In complete analogy to the preceding proof, with $B, A$ replaced by

$$
\mathcal{B}=\left(\begin{array}{cc}
A & B \\
B^{*} & 0
\end{array}\right) \quad \text { and } \mathcal{A}=\left(\begin{array}{cc}
A & 0 \\
0 & \beta^{2} I
\end{array}\right)
$$

respectively, the inf-sup constant is the square root of the smallest eigenvalue $\mu$ of

$$
\mathcal{B}^{*} \mathcal{A}^{-1} \mathcal{B} \mathcal{V}=\mu \mathcal{A} \mathcal{V}
$$


Written out explicitly, with $\mathcal{V}^{T}=(\mathbf{u}, p)^{T}$, this is

$$
\left(\begin{array}{cc}
A & B \\
B^{*} & 0
\end{array}\right)\left(\begin{array}{cc}
A^{-1} & 0 \\
0 & \beta^{-2} I
\end{array}\right)\left(\begin{array}{cc}
A & B \\
B^{*} & 0
\end{array}\right)\left(\begin{array}{l}
\mathbf{u} \\
p
\end{array}\right)=\mu\left(\begin{array}{cc}
A & 0 \\
0 & \beta^{2} I
\end{array}\right)\left(\begin{array}{l}
\mathbf{u} \\
p
\end{array}\right) .
$$

From here we see that $\mu=\nu^{2}$, where $\nu$ is the eigenvalue to

$$
\left(\begin{array}{cc}
A & B \\
B^{*} & 0
\end{array}\right)\left(\begin{array}{l}
\mathbf{u} \\
p
\end{array}\right)=\nu\left(\begin{array}{cc}
A & 0 \\
0 & \beta^{2} I
\end{array}\right)\left(\begin{array}{l}
\mathbf{u} \\
p
\end{array}\right) \text {. }
$$

Explicitly,

$$
\begin{aligned}
A \mathbf{u}+B p & =\nu A \mathbf{u}, \\
B^{*} \mathbf{u} & =\beta^{2} \nu p .
\end{aligned}
$$

From here, we see that $\nu=1$ is an eigenvalue. Suppose next that $\nu \neq 1$. Solving for $(\nu-1) \mathbf{u}$ in the first equation and substituting in the second one gives

$$
B^{*} A^{-1} B p=\beta^{2} \nu(\nu-1) p .
$$

Comparing with (A.3) shows that

$$
\nu(\nu-1)=1,
$$

giving

$$
\nu=\frac{1 \pm \sqrt{5}}{2} .
$$

The constant in the stability condition is thus $\min |\nu|$, i.e.

$$
\min \left\{1, \frac{\sqrt{5}+1}{2}, \frac{\sqrt{5}-1}{2}\right\}=\frac{\sqrt{5}-1}{2}
$$

This completes the proof.

\section{B Equilibration for higher-order conforming and conforming stabilized finite element methods on dual meshes}

This appendix concerns conforming and conforming stabilized finite element methods of Section 7.2. More precisely, for higher-order continuous pressure elements of Section 7.2.2, we show how to, from (7.29), obtain new normal flux functions $\boldsymbol{\Upsilon}_{F}\left(\mathbf{u}_{h}, p_{h}\right)$ for which (7.30) holds. This can be seen as an equivalent of the equilibration procedure of [4] on dual meshes.

Let $D \in \mathcal{D}_{h}^{\text {int }}, V$ be the associated vertex, $T \in \mathfrak{T}_{V}$, and $i=1, \ldots, d$. Denote the contribution to the correction terms of the right-hand side of (7.29) by

$$
\begin{aligned}
m_{V, T, i} & :=-\left(\mathbf{f}+\Delta \mathbf{u}_{h}-\nabla p_{h}, \mathbf{e}_{i}\right)_{T \cap D}+\left(\mathbf{f}+\Delta \mathbf{u}_{h}-\nabla p_{h}, \boldsymbol{\psi}_{V, i}\right)_{T} \\
& -\frac{1}{2} \sum_{F \in \mathcal{F}_{T}^{\text {int }}}\left\langle\llbracket \nabla \mathbf{u}_{h} \mathbf{n}_{F} \rrbracket, \boldsymbol{\psi}_{V, i}\right\rangle_{F}+\frac{1}{2} \sum_{F \in \mathcal{F}_{T}^{\text {int }}}\left\langle\llbracket \nabla \mathbf{u}_{h} \mathbf{n}_{F} \rrbracket, \mathbf{e}_{i}\right\rangle_{F \cap D} .
\end{aligned}
$$

We will speak about these quantities as of "normal fluxes" $m_{V, T, i}$. Remark that $\llbracket \nabla \mathbf{u}_{h} \mathbf{n}_{F} \rrbracket=0$ on such sides $F \in \partial \mathcal{S}_{D}^{\text {int }}$ which are not contained in $\partial \mathcal{T}_{h}$, cf. Figure 7.1. Thus, from (7.29) and the above formula, we have

$$
\begin{aligned}
& \sum_{F \in \mathcal{F}_{D}}\left\langle\boldsymbol{\Upsilon}_{F}\left(\mathbf{u}_{h}\right) \mathbf{n}_{D} \cdot \mathbf{n}_{F}, \mathbf{e}_{i}\right\rangle_{F}-\left(\nabla p_{h}, \mathbf{e}_{i}\right)_{D}+\left(\mathbf{f}, \mathbf{e}_{i}\right)_{D}+\sum_{T \in \mathfrak{T}_{V}} m_{V, T, i}=0, \\
& \quad i=1, \ldots, d, \quad \forall D \in \mathcal{D}_{h}^{\mathrm{int}} .
\end{aligned}
$$




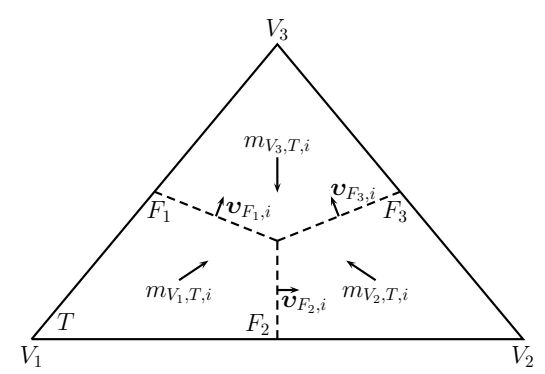

Fig. B.1 Equilibration of the correction terms inside each triangle

For the sake of simplicity, let us define $m_{V, T, i}$ in the same way also for $D \in \mathcal{D}_{h}^{\text {ext }}$ and the associated vertex $V$.

Consider a fixed $T \in \mathcal{T}_{h}$ and $i=1, \ldots, d$. We have associated the normal flux $m_{V_{j}, T, i}$ to each of the vertices $V_{j}$ of $T, j=1, \ldots, d+1$, cf. Figure B.1. We now want to equilibrate the normal fluxes $m_{V_{j}, T, i}$ : the purpose is to associate to each of the sides $F_{m} \subset T, m=1, \ldots, d+1, F_{m} \in \partial \mathcal{S}_{h}^{\text {int }}$ such that $F \subset \partial D$ for some $D \in \mathcal{D}_{h}$, a correction normal flux $v_{F_{m}, i}$ (in the direction of the fixed normal $\mathbf{n}_{F}$ ) such that the following holds (we give an example for $d=2$, corresponding to Figure B.1):

$$
\left(\begin{array}{rrr}
1 & 1 & 0 \\
0 & -1 & 1 \\
-1 & 0 & -1
\end{array}\right)\left(\begin{array}{l}
v_{F_{1}, i} \\
v_{F_{2}, i} \\
v_{F_{3}, i}
\end{array}\right)=\left(\begin{array}{l}
m_{V_{1}, T, i} \\
m_{V_{2}, T, i} \\
m_{V_{3}, T, i}
\end{array}\right) .
$$

The value $m_{V_{1}, T, i}$ represents the total normal flux from the element $T \cap D_{1}$ to the elements $T \cap D_{2}$ and $T \cap D_{3}$ (where $D_{i}$ are the dual volumes associated with the vertices $V_{i}$ ). We clearly want to keep this total normal flux but to split it into the side normal fluxes $v_{F_{1}, i}$ and $v_{F_{2}, i}$; we proceed similarly for $m_{V_{2}, T, i}$ and $m_{V_{3}, T, i}$. The essential feature is that the corrections normal fluxes $v_{F_{m}, i}$ are univocally defined for each side $F_{m}, m=1, \ldots, d+1$, cf. once again Figure B.1.

It turns out that the system matrix in (B3) is singular, as the sum of all the row vectors equals zero. It is, however, easy to check that its rank is equal to $d$. Fortunately, the right-hand side in (B3) is compatible: by the fact that the basis functions $\boldsymbol{\psi}_{V_{j}, i}$ form a partition of unity on the chosen element $T \in \mathcal{T}_{h}$,

$$
\left.\sum_{j=1}^{d+1} \psi_{V_{j}, i}\right|_{T}=\left.\mathbf{e}_{i}\right|_{T}
$$

we easily get

$$
\sum_{j=1}^{d+1} m_{V_{j}, T, i}=0
$$

$i=1, \ldots, d$. Thus, there exists a solution to (B3). Note that (B3) is always a system of a fixed small size $(d+1) \times(d+1)$ on each $T \in \mathcal{T}_{h}$, for approximations (7.11a)$(7.11 \mathrm{~b})$ or $(7.12 \mathrm{a})-(7.12 \mathrm{~b})$ of any order $k$.

Using $v_{F_{m}, i}$ for each $T \in \mathcal{T}_{h}$, we can now define new normal flux functions $\boldsymbol{\Upsilon}_{F}\left(\mathbf{u}_{h}, p_{h}\right)$ for sides $F \in \partial \mathcal{S}_{h}^{\text {int }}$ such that $F \subset \partial D$ for some $D \in \mathcal{D}_{h}$, in a way that (7.30) holds. More precisely, let

$$
\left(\boldsymbol{v}_{F}\left(\mathbf{u}_{h}, p_{h}\right)\right)^{i}:=|F|^{-1} v_{F, i}, \quad i=1, \ldots, d .
$$

Note that, consequently, (B3) gives

$$
\sum_{T \in \mathfrak{T}_{V}} m_{V, T, i}=\sum_{F \in \mathcal{F}_{D}} v_{F, i} \mathbf{n}_{D} \cdot \mathbf{n}_{F}=\sum_{F \in \mathcal{F}_{D}}\left\langle\boldsymbol{v}_{F}\left(\mathbf{u}_{h}, p_{h}\right) \mathbf{n}_{D} \cdot \mathbf{n}_{F}, \mathbf{e}_{i}\right\rangle_{F}
$$

for every $D \in \mathcal{D}_{h}^{\text {int }}$ and the associated vertex $V, i=1, \ldots, d$. Let $F \in \partial \mathcal{S}_{h}^{\text {int }}$ such that $F \subset \partial D$ for some $D \in \mathcal{D}_{h}$ and set

$$
\boldsymbol{\Upsilon}_{F}\left(\mathbf{u}_{h}, p_{h}\right):=\left.\left(\nabla \mathbf{u}_{h} \mathbf{n}_{F}\right)\right|_{F}+\boldsymbol{v}_{F}\left(\mathbf{u}_{h}, p_{h}\right)
$$


We then see that (B2) together with (B5) and (B6) implies (7.30).

Acknowledgements We are indebted to Prof. Jean-François Maître (Ecole Centrale de Lyon) for showing us Lemma 3.1.

\section{References}

1. Achdou, Y., Bernardi, C., And Coquel, F. A priori and a posteriori analysis of finite volume discretizations of Darcy's equations. Numer. Math. 96, 1 (2003), 17-42.

2. Ainsworth, M. A synthesis of a posteriori error estimation techniques for conforming, non-conforming and discontinuous Galerkin finite element methods. In Recent advances in adaptive computation, vol. 383 of Contemp. Math. Amer. Math. Soc., Providence, RI, 2005, pp. 1-14.

3. Ainsworth, M. A posteriori error estimation for discontinuous Galerkin finite element approximation. SIAM J. Numer. Anal. 45, 4 (2007), 1777-1798.

4. Ainsworth, M., And Oden, J. T. A posteriori error estimation in finite element analysis. Pure and Applied Mathematics (New York). Wiley-Interscience [John Wiley \& Sons], New York, 2000.

5. Arbogast, T., And Chen, Z. On the implementation of mixed methods as nonconforming methods for second-order elliptic problems. Math. Comp. 64, 211 (1995), 943-972.

6. Arnold, D. N., And Brezzi, F. Mixed and nonconforming finite element methods: implementation, postprocessing and error estimates. RAIRO Modél. Math. Anal. Numér. 19, 1 (1985), 7-32.

7. Arnold, D. N., Brezzi, F., AND Fortin, M. A stable finite element for the Stokes equations. Calcolo 21, 4 (1984), 337-344 (1985).

8. Bank, R. E., And Rose, D. J. Some error estimates for the box method. SIAM J. Numer. Anal. 24, 4 (1987), 777-787.

9. Bebendorf, M. A note on the Poincaré inequality for convex domains. $Z$. Anal. Anwendungen 22, 4 (2003), 751-756.

10. Becker, R., Capatina, D., And Joie, J. A dG method for the Stokes equations related to nonconforming approximations. HAL preprint 00380772, 2009.

11. Beirão da Veiga, L., Gyrya, V.. Lipnikov, K., and Manzini, G. Mimetic finite difference method for the Stokes problem on polygonal meshes. J. Comp. Phys. 228, 19 (2009), 7215-7232.

12. Bercovier, M., and Pironneau, O. Error estimates for finite element method solution of the Stokes problem in the primitive variables. Numer. Math. 33, 2 (1979), 211-224.

13. Braess, D., Pillwein, V., And SchöBerl, J. Equilibrated residual error estimates are p-robust. Comput. Methods Appl. Mech. Engrg. 198, 13-14 (2009), 1189-1197.

14. Braess, D., AND SchöBerl, J. Equilibrated residual error estimator for edge elements. Math. Comp. 77, 262 (2008), 651-672.

15. Brezzi, F., And Douglas, Jr., J. Stabilized mixed methods for the Stokes problem. Numer. Math. 53, 1-2 (1988), 225-235.

16. Brezzi, F., AND FAlK, R. S. Stability of higher-order Hood-Taylor methods. SIAM J. Numer. Anal. 28, 3 (1991), 581-590.

17. Brezzi, F., AND Fortin, M. Mixed and hybrid finite element methods, vol. 15 of Springer Series in Computational Mathematics. Springer-Verlag, New York, 1991.

18. Brezzi, F., And PitKäranta, J. On the stabilization of finite element approximations of the Stokes equations. In Efficient solutions of elliptic systems (Kiel, 1984), vol. 10 of Notes Numer. Fluid Mech. Vieweg, Braunschweig, 1984, pp. 11-19.

19. Burman, E., AND ERn, A. Continuous interior penalty $h p$-finite element methods for advection and advection-diffusion equations. Math. Comp. 76, 259 (2007), 1119-1140.

20. Carstensen, C. A unifying theory of a posteriori finite element error control. Numer. Math. 100, 4 (2005), 617-637.

21. Carstensen, C., Gedicke, J., and PARK, E.-J. Arnold-Winther mixed finite elements for the Stokes problem. Submitted to SIAM J. Sci. Comput., 2010. 
22. Carstensen, C., Gudi, T., And Jensen, M. A unifying theory of a posteriori error control for discontinuous Galerkin FEM. Numer. Math. 112, 3 (2009), 363-379.

23. Carstensen, C., And Hu, J. A unifying theory of a posteriori error control for nonconforming finite element methods. Numer. Math. 107, 3 (2007), 473-502.

24. Cheddadi, I., Fučík, R., Prieto, M. I., And Vohralík, M. Guaranteed and robust a posteriori error estimates for singularly perturbed reaction-diffusion problems. M2AN Math. Model. Numer. Anal. 43, 5 (2009), 867-888.

25. Crouzeix, M., and Raviart, P.-A. Conforming and nonconforming finite element methods for solving the stationary Stokes equations. I. Rev. Française Automat. Informat. Recherche Opérationnelle Sér. Rouge 7, R-3 (1973), 33-75.

26. DARI, E., Durán, R., AND PADra, C. Error estimators for nonconforming finite element approximations of the Stokes problem. Math. Comp. 64, 211 (1995), 1017-1033.

27. Destuynder, P., And Métivet, B. Explicit error bounds in a conforming finite element method. Math. Comp. 68, 228 (1999), 1379-1396.

28. Dobrowolski, M. On the LBB condition in the numerical analysis of the Stokes equations. Appl. Numer. Math. 54, 3-4 (2005), 314-323.

29. Dörfler, W., AND Ainsworth, M. Reliable a posteriori error control for nonconformal finite element approximation of Stokes flow. Math. Comp. 74, 252 (2005), 1599-1619.

30. Droniou, J., And Eymard, R. Study of the mixed finite volume method for Stokes and Navier-Stokes equations. Numer. Methods Partial Differential Equations 25, 1 (2009), 137-171.

31. Ern, A., Nicaise, S., And Vohralík, M. An accurate $\mathbf{H}($ div) flux reconstruction for discontinuous Galerkin approximations of elliptic problems. $C$. R. Math. Acad. Sci. Paris 345, 12 (2007), 709-712.

32. Ern, A., Stephansen, A. F., And Vohralík, M. Guaranteed and robust discontinuous Galerkin a posteriori error estimates for convection-diffusionreaction problems. J. Comput. Appl. Math. 234, 1 (2010), 114-130.

33. ERn, A., AND Vohralík, M. Flux reconstruction and a posteriori error estimation for discontinuous Galerkin methods on general nonmatching grids. $C$. R. Math. Acad. Sci. Paris 347, 7-8 (2009), 441-444.

34. ERn, A., AND Vohralík, M. A posteriori error estimation based on potential and flux reconstruction for the heat equation. SIAM J. Numer. Anal. 48, 1 (2010), 198-223.

35. Franca, L. P., Hughes, T. J. R. and Stenberg, R. Stabilized finite element methods. In Incompressible computational fluid dynamics: trends and advances. Cambridge Univ. Press, Cambridge, 1993, Reprint 2008, pp. 87-107.

36. Franca, L. P., And Stenberg, R. Error analysis of Galerkin least squares methods for the elasticity equations. SIAM J. Numer. Anal. 28, 6 (1991), $1680-1697$.

37. Girault, V., And Raviart, P.-A. Finite element methods for Navier-Stokes equations, vol. 5 of Springer Series in Computational Mathematics. SpringerVerlag, Berlin, 1986. Theory and algorithms.

38. Glowinski, R., And Pironneau, O. On numerical methods for the Stokes problem. In Energy methods in finite element analysis. Wiley, Chichester, 1979, pp. 243-264.

39. HlaváČEK, I., Haslinger, J., NeČas, J., And LovíšEK, J. Solution of variational inequalities in mechanics, vol. 66 of Applied Mathematical Sciences. Springer-Verlag, New York, 1988. Translated from the Slovak by J. Jarník.

40. Houston, P., Schötzau, D., And Wihler, T. P. Energy norm a posteriori error estimation for mixed discontinuous Galerkin approximations of the Stokes problem. J. Sci. Comput. 22/23 (2005), 347-370.

41. Hughes, T. J. R., Franca, L. P., and Balestra, M. A new finite element formulation for computational fluid dynamics. V. Circumventing the BabuškaBrezzi condition: a stable Petrov-Galerkin formulation of the Stokes problem accommodating equal-order interpolations. Comput. Methods Appl. Mech. Engrg. 59, 1 (1986), 85-99.

42. Karakashian, O. A., And Pascal, F. A posteriori error estimates for a discontinuous Galerkin approximation of second-order elliptic problems. SIAM J. Numer. Anal. 41, 6 (2003), 2374-2399.

43. KIM, K. Y. A posteriori error analysis for locally conservative mixed methods. Math. Comp. 76, 257 (2007), 43-66.

44. Ladevìze, P., And Leguillon, D. Error estimate procedure in the finite element method and applications. SIAM J. Numer. Anal. 20, 3 (1983), 485509 . 
45. Luce, R., And Wohlmuth, B. I. A local a posteriori error estimator based on equilibrated fluxes. SIAM J. Numer. Anal. 42, 4 (2004), 1394-1414.

46. Mâ̂tre, J.-F. Personnal communication. 2010.

47. Payne, L. E., And Weinberger, H. F. An optimal Poincaré inequality for convex domains. Arch. Rational Mech. Anal. 5 (1960), 286-292.

48. Pironneau, O. Finite element methods for fluids. John Wiley \& Sons Ltd., Chichester, 1989. Translated from the French.

49. Prager, W., And Synge, J. L. Approximations in elasticity based on the concept of function space. Quart. Appl. Math. 5 (1947), 241-269.

50. REPIN, S. I. A posteriori error estimation for nonlinear variational problems by duality theory. Zap. Nauchn. Sem. S.-Peterburg. Otdel. Mat. Inst. Steklov. (POMI) 243, Kraev. Zadachi Mat. Fiz. i Smezh. Vopr. Teor. Funktsii. 28 (1997), 201-214, 342 .

51. Repin, S. I., And Stenberg, R. A posteriori error estimates for the generalized Stokes problem. J. Math. Sci. (N. Y.) 142, 1 (2007), 1828-1843. Problems in mathematical analysis. No. 34.

52. Scott, L. R., And Vogelius, M. Conforming finite element methods for incompressible and nearly incompressible continua. In Large-scale computations in fluid mechanics, Part 2 (La Jolla, Calif., 1983), vol. 22 of Lectures in Appl. Math. Amer. Math. Soc., Providence, RI, 1985, pp. 221-244.

53. Stenberg, R. Some new families of finite elements for the Stokes equations. Numer. Math. 56, 8 (1990), 827-838.

54. TAYlOR, C., AND HoOd, P. A numerical solution of the Navier-Stokes equations using the finite element technique. Internat. J. Comput. $\&$ Fluids 1, 1 (1973), 73-100.

55. VERFÜRTH, R. A combined conjugate gradient-multigrid algorithm for the numerical solution of the Stokes problem. IMA J. Numer. Anal. 4, 4 (1984), $441-455$.

56. Verfürth, R. A posteriori error estimators for the Stokes equations. Numer. Math. 55, 3 (1989), 309-325.

57. Verfürth, R. A posteriori error estimators for the Stokes equations. II. Nonconforming discretizations. Numer. Math. 60, 2 (1991), 235-249.

58. Vohralík, M. On the discrete Poincaré-Friedrichs inequalities for nonconforming approximations of the Sobolev space $H^{1}$. Numer. Funct. Anal. Optim. 26, 7-8 (2005), 925-952.

59. Vohralík, M. A posteriori error estimates for lowest-order mixed finite element discretizations of convection-diffusion-reaction equations. SIAM J. $\mathrm{Nu}$ mer. Anal. 45, 4 (2007), 1570-1599.

60. Vohralík, M. A posteriori error estimation in the conforming finite element method based on its local conservativity and using local minimization. $C$. $R$. Math. Acad. Sci. Paris 346, 11-12 (2008), 687-690.

61. Vohralík, M. Residual flux-based a posteriori error estimates for finite volume and related locally conservative methods. Numer. Math. 111, 1 (2008), 121158.

62. Vohralík, M. Unified primal formulation-based a priori and a posteriori error analysis of mixed finite element methods. Math. Comp. 79, 272 (2010), 20012032.

63. Vohralík, M. Guaranteed and fully robust a posteriori error estimates for conforming discretizations of diffusion problems with discontinuous coefficients. J. Sci. Comput. 46 (2011), 397-438.

64. WANG, J., WAND, Y., AND YE, X. A unified posteriori error estimator for finite element methods for the Stokes equations. Submitted to SIAM J. Numer. Anal., 2010. 